\title{
Compact embeddings in Besov-type and Triebel-Lizorkin-type spaces on bounded domains
}

\author{
Helena F. Gonçalves ${ }^{1}$ (D) - Dorothee D. Haroske ${ }^{1}$ (D) $\cdot$ Leszek Skrzypczak $^{2}$
}

Received: 7 January 2020 / Accepted: 22 June 2020 / Published online: 16 July 2020

(c) The Author(s) 2020

\section{Abstract}

We study embeddings of Besov-type and Triebel-Lizorkin-type spaces, $\operatorname{id}_{\tau}: B_{p_{1}, q_{1}}^{s_{1}, \tau_{1}}(\Omega)$ $\hookrightarrow B_{p_{2}, q_{2}}^{s_{2}, \tau_{2}}(\Omega)$ and $\mathrm{id}_{\tau}: F_{p_{1}, q_{1}}^{s_{1}, \tau_{1}}(\Omega) \hookrightarrow F_{p_{2}, q_{2}}^{s_{2}, \tau_{2}}(\Omega)$, where $\Omega \subset \mathbb{R}^{d}$ is a bounded domain, and obtain necessary and sufficient conditions for the compactness of $\mathrm{id}_{\tau}$. Moreover, we characterize its entropy and approximation numbers. Surprisingly, these results are completely obtained via embeddings and the application of the corresponding results for classical Besov and Triebel-Lizorkin spaces as well as for Besov-Morrey and Triebel-Lizorkin-Morrey spaces.

Keywords Besov-type spaces · Triebel-Lizorkin-type spaces · Compact embeddings · Entropy numbers · Approximation numbers

Mathematics Subject Classification 46E35 - 41A46

\section{Introduction}

Usually called smoothness spaces of Morrey type or, for short, smoothness Morrey spaces, these function spaces are built upon Morrey spaces $\mathcal{M}_{u, p}\left(\mathbb{R}^{d}\right), 0<p \leq u<$

Helena F. Gonçalves, Dorothee D. Haroske and Leszek Skrzypczak were partially supported by the German Research Foundation (DFG), Grant No. Ha 2794/8-1. Leszek Skrzypczak was also supported by National Science Center, Poland, Grant No. 2013/10/A/ST1/00091.

$凶 \quad$ Helena F. Gonçalves

helena.goncalves@uni-jena.de

Dorothee D. Haroske

dorothee.haroske@uni-jena.de

Leszek Skrzypczak

1skrzyp@amu.edu.pl

1 Institute of Mathematics, Friedrich-Schiller-University Jena, 07737 Jena, Germany

2 Faculty of Mathematics and Computer Science, Adam Mickiewicz University, ul. Uniwersytetu Poznańskiego 4, 61-614 Poznan, Poland 
$\infty$, and attracted some attention in the last decades, motivated firstly by possible applications. They include Besov-Morrey spaces $\mathcal{N}_{u, p, q}^{s}\left(\mathbb{R}^{d}\right)$, Triebel-Lizorkin-Morrey spaces $\mathcal{E}_{u, p, q}^{s}\left(\mathbb{R}^{d}\right), 0<p \leq u<\infty, 0<q \leq \infty, s \in \mathbb{R}$, Besov-type spaces $B_{p, q}^{s, \tau}\left(\mathbb{R}^{d}\right)$ and Triebel-Lizorkin-type spaces $F_{p, q}^{s, \tau}\left(\mathbb{R}^{d}\right), 0<p<\infty, 0<q \leq \infty$, $\tau \geq 0, s \in \mathbb{R}$.

The classical Morrey spaces $\mathcal{M}_{u, p}, 0<p \leq u<\infty$, were introduced by Morrey [22] and are part of a wider class of Morrey-Campanato spaces, cf. [23]. They can be seen as a complement to $L_{p}$ spaces, since $\mathcal{M}_{p, p}\left(\mathbb{R}^{d}\right)=L_{p}\left(\mathbb{R}^{d}\right)$.

The Besov-Morrey spaces $\mathcal{N}_{u, p, q}^{s}\left(\mathbb{R}^{d}\right)$ were introduced by Kozono and Yamazaki [19] and used by them and later on by Mazzucato [21] in the study of Navier-Stokes equations. In [34], Tang and $\mathrm{Xu}$ introduced the corresponding Triebel-Lizorkin-Morrey spaces $\mathcal{E}_{u, p, q}^{s}\left(\mathbb{R}^{d}\right)$, thanks to establishing the Morrey version of Fefferman-Stein vector-valued inequality. Some properties of these spaces including their wavelet characterizations were later described in the papers by Sawano [27,28], Sawano and Tanaka [29,30] and Rosenthal [26]. Recently, some limiting embedding properties of these spaces were investigated in a series of papers [13-16].

Another class of generalizations, the Besov-type space $B_{p, q}^{s, \tau}\left(\mathbb{R}^{d}\right)$ and the TriebelLizorkin-type space $F_{p, q}^{s, \tau}\left(\mathbb{R}^{d}\right)$ were introduced in [45]. Their homogeneous versions were originally investigated by El Baraka [8-10] and by Yuan and Yang [40,41]. There are also some applications in partial differential equations for spaces of type $B_{p, q}^{s, \tau}\left(\mathbb{R}^{d}\right)$ and $F_{p, q}^{s, \tau}\left(\mathbb{R}^{d}\right)$, such as (fractional) Navier-Stokes equations, cf. [20].

Although the above scales are defined in different ways, they share some properties and are related to each other by a number of embeddings and coincidences. For instance, they both include the classical spaces of type $B_{p, q}^{s}\left(\mathbb{R}^{d}\right)$ and $F_{p, q}^{s}\left(\mathbb{R}^{d}\right)$ as special cases. We refer to our papers mentioned above, to the recently published papers $[43,44]$, but in particular to the fine surveys $[32,33]$ by Sickel.

There is still a third approach, due to Triebel, who introduced and studied in [38] local spaces and in [39] hybrid spaces, together with their use in heat equations and Navier-Stokes equations. However, since the hybrid spaces coincide with appropriately chosen spaces of type $B_{p, q}^{s, \tau}\left(\mathbb{R}^{d}\right)$ or $F_{p, q}^{s, \tau}\left(\mathbb{R}^{d}\right)$, respectively, cf. [46], we do not have to deal with them separately now.

In this paper we investigate the compactness of the embeddings of the spaces $B_{p, q}^{s, \tau}(\Omega)$ and $F_{p, q}^{s, \tau}(\Omega)$, where $\Omega \subset \mathbb{R}^{d}$ is a bounded domain, i.e. a bounded open set in $\mathbb{R}^{d}$. In particular, our first goal is to find necessary and sufficient conditions for the compactness of the embeddings

$$
\operatorname{id}_{\tau}: A_{p_{1}, q_{1}}^{s_{1}, \tau_{1}}(\Omega) \hookrightarrow A_{p_{2}, q_{2}}^{s_{2}, \tau_{2}}(\Omega),
$$

where $A=B$ or $A=F$, cf. Theorem 3.2. Here we prove that $\mathrm{id}_{\tau}$ is compact if, and only if,

$$
\frac{s_{1}-s_{2}}{d}>\max \left\{\left(\tau_{2}-\frac{1}{p_{2}}\right)_{+}-\left(\tau_{1}-\frac{1}{p_{1}}\right)_{+}, \frac{1}{p_{1}}-\tau_{1}-\min \left\{\frac{1}{p_{2}}-\tau_{2}, \frac{1}{p_{2}}\left(1-p_{1} \tau_{1}\right)_{+}\right\}\right\},
$$


where we use the notation $a_{+}:=\max \{a, 0\}$. At this point, this work can be seen as a counterpart of the papers [14-16], where we studied the compactness of the corresponding embeddings of the spaces $\mathcal{N}_{u, p, q}^{s}$ and $\mathcal{E}_{u, p, q}^{s}$.

Usually one would start by studying the continuity of such embeddings and later proceed to the compactness. Here we do it differently and start by dealing with the compactness. Our technique relies basically on embeddings. Since for compactness one always has strict inequalities, like condition (1.2), one can always have further embeddings in between the considered spaces. Therefore, we take advantage of the relations between this scale, the smoothness Morrey spaces $\mathcal{N}_{u, p, q}^{s}$ and $\mathcal{E}_{u, p, q}^{s}$ and the classical spaces of type $B_{p, q}^{s}$ and $F_{p, q}^{s}$, and use the corresponding results for these spaces to obtain our main result.

Afterwards we qualify the compactness of $\mathrm{id}_{\tau}$ in (1.1) by means of entropy and approximation numbers. In the recent works [16,17], we characterised entropy and approximation numbers of the embedding

$$
\operatorname{id}_{\mathcal{A}}: \mathcal{A}_{u_{1}, p_{1}, q_{1}}^{s_{1}}(\Omega) \hookrightarrow \mathcal{A}_{u_{2}, p_{2}, q_{2}}^{s_{2}}(\Omega),
$$

with $\mathcal{A}=\mathcal{N}$ or $\mathcal{A}=\mathcal{E}$. However, to the best of our knowledge, apart from a result obtained in [43] for approximation numbers when the target space is $L_{\infty}$, nothing is known on this matter for embeddings between spaces of type $A_{p, q}^{s, \tau}$. Here we contribute a little more to the development of this topic, establishing some partial counterparts of the results proved in [17].

This paper is organized as follows. In Sect. 2 we present and collect some basic facts about smoothness Morrey spaces, on $\mathbb{R}^{d}$ and on bounded domains $\Omega \subset \mathbb{R}^{d}$, and introduce the notions of entropy and approximation numbers. In Sect. 3 we are concerned with the compactness of the above-described embeddings of Besov-type and Triebel-Lizorkin-type spaces on bounded domains. We also prove an extension of the results obtained in [14] for the scale $\mathcal{N}_{u, p, q}^{s}$ to the cases when $p_{i}=u_{i}=\infty$, $i=1,2$. Moreover, we collect some immediate consequences of the main result, when we consider particular source and/or target spaces. In Sect. 4 we end up by characterizing entropy and approximation numbers of the embedding $\mathrm{id}_{\tau}$ in (1.1), collecting also some special cases.

\section{Preliminaries}

First we fix some notation. By $\mathbb{N}$ we denote the set of natural numbers, by $\mathbb{N}_{0}$ the set $\mathbb{N} \cup\{0\}$, and by $\mathbb{Z}^{d}$ the set of all lattice points in $\mathbb{R}^{d}$ having integer components. For $a \in \mathbb{R}$, let $a_{+}:=\max \{a, 0\}$. All unimportant positive constants will be denoted by $C$, occasionally with subscripts. By the notation $A \lesssim B$, we mean that there exists a positive constant $C$ such that $A \leq C B$, whereas the symbol $A \sim B$ stands for $A \lesssim B \lesssim A$. We denote by $B(x, r):=\left\{y \in \mathbb{R}^{d}:|x-y|<r\right\}$ the ball centred at $x \in \mathbb{R}^{d}$ with radius $r>0$, and $|\cdot|$ denotes the Lebesgue measure when applied to measurable subsets of $\mathbb{R}^{d}$.

Given two (quasi-)Banach spaces $X$ and $Y$, we write $X \hookrightarrow Y$ if $X \subset Y$ and the natural embedding of $X$ into $Y$ is continuous. 


\subsection{Smoothness spaces of Morrey type on $\mathbb{R}^{d}$}

Let $\mathcal{S}\left(\mathbb{R}^{d}\right)$ be the set of all Schwartz functions on $\mathbb{R}^{d}$, endowed with the usual topology, and denote by $\mathcal{S}^{\prime}\left(\mathbb{R}^{d}\right)$ its topological dual, namely, the space of all bounded linear functionals on $\mathcal{S}\left(\mathbb{R}^{d}\right)$ endowed with the weak $*$-topology. For all $f \in \mathcal{S}\left(\mathbb{R}^{d}\right)$ or $\mathcal{S}^{\prime}\left(\mathbb{R}^{d}\right)$, we use $\widehat{f}$ to denote its Fourier transform, and $f^{\vee}$ for its inverse. Let $\mathcal{Q}$ be the collection of all dyadic cubes in $\mathbb{R}^{d}$, namely, $\mathcal{Q}:=\left\{Q_{j, k}:=2^{-j}\left([0,1)^{d}+\right.\right.$ $\left.k): j \in \mathbb{Z}, k \in \mathbb{Z}^{d}\right\}$. The symbol $\ell(Q)$ denotes the side-length of the cube $Q$ and $j_{Q}:=-\log _{2} \ell(Q)$.

Let $\varphi_{0}, \varphi \in \mathcal{S}\left(\mathbb{R}^{d}\right)$ be such that

$$
\operatorname{supp} \widehat{\varphi_{0}} \subset\left\{\xi \in \mathbb{R}^{d}:|\xi| \leq 2\right\}, \quad\left|\widehat{\varphi_{0}}(\xi)\right| \geq C \text { if }|\xi| \leq 5 / 3
$$

and

$$
\operatorname{supp} \widehat{\varphi} \subset\left\{\xi \in \mathbb{R}^{d}: 1 / 2 \leq|\xi| \leq 2\right\} \text { and }|\widehat{\varphi}(\xi)| \geq C \text { if } 3 / 5 \leq|\xi| \leq 5 / 3
$$

where $C$ is a positive constant. In what follows, for all $\varphi \in \mathcal{S}\left(\mathbb{R}^{d}\right)$ and $j \in \mathbb{N}$, $\varphi_{j}(\cdot):=2^{j d} \varphi\left(2^{j} \cdot\right)$.

Definition 2.1 Let $s \in \mathbb{R}, \tau \in[0, \infty), q \in(0, \infty]$ and $\varphi_{0}, \varphi \in \mathcal{S}\left(\mathbb{R}^{d}\right)$ be as in (2.1) and (2.2), respectively.

(i) Let $p \in(0, \infty]$. The Besov-type space $B_{p, q}^{s, \tau}\left(\mathbb{R}^{d}\right)$ is defined to be the collection of all $f \in \mathcal{S}^{\prime}\left(\mathbb{R}^{d}\right)$ such that

$$
\left\|f \mid B_{p, q}^{s, \tau}\left(\mathbb{R}^{d}\right)\right\|:=\sup _{P \in \mathcal{Q}} \frac{1}{|P|^{\tau}}\left\{\sum_{j=\max \left\{j_{P}, 0\right\}}^{\infty} 2^{j s q}\left[\int_{P}\left|\varphi_{j} * f(x)\right|^{p} \mathrm{~d} x\right]^{\frac{q}{p}}\right\}^{\frac{1}{q}}<\infty
$$

with the usual modifications made in case of $p=\infty$ and/or $q=\infty$.

(ii) Let $p \in(0, \infty)$. The Triebel-Lizorkin-type space $F_{p, q}^{s, \tau}\left(\mathbb{R}^{d}\right)$ is defined to be the collection of all $f \in \mathcal{S}^{\prime}\left(\mathbb{R}^{d}\right)$ such that

$$
\left\|f \mid F_{p, q}^{s, \tau}\left(\mathbb{R}^{d}\right)\right\|:=\sup _{P \in \mathcal{Q}} \frac{1}{|P|^{\tau}}\left\{\int_{P}\left[\sum_{j=\max \left\{j_{P}, 0\right\}}^{\infty} 2^{j s q}\left|\varphi_{j} * f(x)\right|^{q}\right]^{\frac{p}{q}} \mathrm{~d} x\right\}^{\frac{1}{p}}<\infty
$$

with the usual modification made in case of $q=\infty$.

Remark 2.2 These spaces were introduced in [45]. To some extent the scale of Nikol'skij-Besov type spaces $B_{p, q}^{s, \tau}\left(\mathbb{R}^{d}\right)$ had already been studied in [8-10].

We shall collect some features of these spaces below, but introduce first another scale of smoothness spaces of Morrey type. Recall first that the Morrey space $\mathcal{M}_{u, p}\left(\mathbb{R}^{d}\right)$, 
$0<p \leq u<\infty$, is defined to be the set of all locally $p$-integrable functions $f \in L_{p}^{\text {loc }}\left(\mathbb{R}^{d}\right)$ such that

$$
\left\|f \mid \mathcal{M}_{u, p}\left(\mathbb{R}^{d}\right)\right\|:=\sup _{x \in \mathbb{R}^{d}, R>0} R^{\frac{d}{u}-\frac{d}{p}}\left[\int_{B(x, R)}|f(y)|^{p} \mathrm{~d} y\right]^{\frac{1}{p}}<\infty .
$$

Remark 2.3 The spaces $\mathcal{M}_{u, p}\left(\mathbb{R}^{d}\right)$ are quasi-Banach spaces (Banach spaces for $p \geq$ 1). They originated from Morrey's study on PDE (see [22]) and are part of the wider class of Morrey-Campanato spaces; cf. [23]. They can be considered as a complement to $L_{p}$ spaces. As a matter of fact, $\mathcal{M}_{p, p}\left(\mathbb{R}^{d}\right)=L_{p}\left(\mathbb{R}^{d}\right)$ with $p \in(0, \infty)$. To extend this relation, we put $\mathcal{M}_{\infty, \infty}\left(\mathbb{R}^{d}\right)=L_{\infty}\left(\mathbb{R}^{d}\right)$. One can easily see that $\mathcal{M}_{u, p}\left(\mathbb{R}^{d}\right)=\{0\}$ for $u<p$, and that for $0<p_{2} \leq p_{1} \leq u<\infty$,

$$
L_{u}\left(\mathbb{R}^{d}\right)=\mathcal{M}_{u, u}\left(\mathbb{R}^{d}\right) \hookrightarrow \mathcal{M}_{u, p_{1}}\left(\mathbb{R}^{d}\right) \hookrightarrow \mathcal{M}_{u, p_{2}}\left(\mathbb{R}^{d}\right)
$$

In an analogous way, one can define the spaces $\mathcal{M}_{\infty, p}\left(\mathbb{R}^{d}\right), p \in(0, \infty)$, but using the Lebesgue differentiation theorem, one can easily prove that $\mathcal{M}_{\infty, p}\left(\mathbb{R}^{d}\right)=L_{\infty}\left(\mathbb{R}^{d}\right)$.

Next we recall the definition of the other scale of smoothness spaces of Morrey type we deal with in this paper.

Definition 2.4 Let $0<p \leq u<\infty$ or $p=u=\infty$. Let $q \in(0, \infty], s \in \mathbb{R}$ and $\varphi_{0}$, $\varphi \in \mathcal{S}\left(\mathbb{R}^{d}\right)$ be as in (2.1) and (2.2), respectively.

(i) The Besov-Morrey space $\mathcal{N}_{u, p, q}^{s}\left(\mathbb{R}^{d}\right)$ is defined to be the set of all distributions $f \in \mathcal{S}^{\prime}\left(\mathbb{R}^{d}\right)$ such that

$$
\left\|f \mid \mathcal{N}_{u, p, q}^{s}\left(\mathbb{R}^{d}\right)\right\|:=\left[\sum_{j=0}^{\infty} 2^{j s q}\left\|\varphi_{j} * f \mid \mathcal{M}_{u, p}\left(\mathbb{R}^{d}\right)\right\|^{q}\right]^{1 / q}<\infty
$$

with the usual modification made in case of $q=\infty$.

(ii) Let $u \in(0, \infty)$. The Triebel-Lizorkin-Morrey space $\mathcal{E}_{u, p, q}^{s}\left(\mathbb{R}^{d}\right)$ is defined to be the set of all distributions $f \in \mathcal{S}^{\prime}\left(\mathbb{R}^{d}\right)$ such that

$$
\left\|f\left|\mathcal{E}_{u, p, q}^{s}\left(\mathbb{R}^{d}\right)\|:=\|\left[\sum_{j=0}^{\infty} 2^{j s q}\left|\left(\varphi_{j} * f\right)(\cdot)\right|^{q}\right]^{1 / q}\right| \mathcal{M}_{u, p}\left(\mathbb{R}^{d}\right)\right\|<\infty
$$

with the usual modification made in case of $q=\infty$.

Remark 2.5 Besov-Morrey spaces were introduced by Kozono and Yamazaki [19]. They studied semi-linear heat equations and Navier-Stokes equations with initial data belonging to Besov-Morrey spaces. The investigations were continued by Mazzucato [21], where one can find the atomic decomposition of the spaces. The TriebelLizorkin-Morrey spaces were later introduced by Tang and Xu [34]. We follow the ideas of Tang and Xu [34], where a somewhat different definition is proposed. The 
ideas were further developed by Sawano and Tanaka [27-30]. The most systematic and general approach to the spaces of this type can be found in the monograph [45] or in the survey papers by Sickel $[32,33]$.

Remark 2.6 Note that for $u=p$ or $\tau=0$ we re-obtain the usual Besov and TriebelLizorkin spaces:

$$
\mathcal{N}_{p, p, q}^{s}\left(\mathbb{R}^{d}\right)=B_{p, q}^{s}\left(\mathbb{R}^{d}\right)=B_{p, q}^{s, 0}\left(\mathbb{R}^{d}\right)
$$

and

$$
\mathcal{E}_{p, p, q}^{s}\left(\mathbb{R}^{d}\right)=F_{p, q}^{s}\left(\mathbb{R}^{d}\right)=F_{p, q}^{s, 0}\left(\mathbb{R}^{d}\right)
$$

where $B_{p, q}^{s}\left(\mathbb{R}^{d}\right)$ and $F_{p, q}^{s}\left(\mathbb{R}^{d}\right)$ denote the classical Besov spaces and Triebel-Lizorkin spaces, respectively. There exists extensive literature on such spaces; we refer, in particular, to the series of monographs [35-37] for a comprehensive treatment.

Convention We adopt the nowadays usual custom to write $A_{p, q}^{s}$ instead of $B_{p, q}^{s}$ or $F_{p, q}^{s}$, $A_{p, q}^{s, \tau}$ instead of $B_{p, q}^{s, \tau}$ or $F_{p, q}^{s, \tau}$, and $\mathcal{A}_{u, p, q}^{s}$ instead of $\mathcal{N}_{u, p, q}^{s}$ or $\mathcal{E}_{u, p, q}^{s}$, respectively, when both scales of spaces are meant simultaneously in some context.

We collect some basic properties of the scales $A_{p, q}^{s, \tau}\left(\mathbb{R}^{d}\right)$ and $\mathcal{A}_{u, p, q}^{s}\left(\mathbb{R}^{d}\right)$. The spaces $A_{p, q}^{s, \tau}\left(\mathbb{R}^{d}\right)$ and $\mathcal{A}_{u, p, q}^{s}\left(\mathbb{R}^{d}\right)$ are independent of the particular choices of $\varphi_{0}, \varphi$ appearing in their definitions. They are quasi-Banach spaces (Banach spaces for $p, q \geq 1$ ), and $\mathcal{S}\left(\mathbb{R}^{d}\right) \hookrightarrow \mathcal{A}_{u, p, q}^{s}\left(\mathbb{R}^{d}\right), A_{p, q}^{s, \tau}\left(\mathbb{R}^{d}\right) \hookrightarrow \mathcal{S}^{\prime}\left(\mathbb{R}^{d}\right)$. In case of $\tau<0$ or $u<p$ we have $A_{p, q}^{s, \tau}\left(\mathbb{R}^{d}\right)=\mathcal{A}_{u, p, q}^{s}\left(\mathbb{R}^{d}\right)=\{0\}$.

Next we recall some basic embeddings results needed in the sequel. We refer to the references given above. For the spaces $A_{p, q}^{s, \tau}\left(\mathbb{R}^{d}\right)$ it is known that

$$
A_{p, r}^{s+\varepsilon}\left(\mathbb{R}^{d}\right) \hookrightarrow A_{p, q}^{s, \tau}\left(\mathbb{R}^{d}\right) \quad \text { if } \quad \varepsilon \in(0, \infty), \quad r, q \in(0, \infty],
$$

and

$$
A_{p, q_{1}}^{s, \tau}\left(\mathbb{R}^{d}\right) \hookrightarrow A_{p, q_{2}}^{s, \tau}\left(\mathbb{R}^{d}\right) \quad \text { if } \quad q_{1} \leq q_{2},
$$

as well as

$$
B_{p, \min \{p, q\}}^{s, \tau}\left(\mathbb{R}^{d}\right) \hookrightarrow F_{p, q}^{s, \tau}\left(\mathbb{R}^{d}\right) \hookrightarrow B_{p, \max \{p, q\}}^{s, \tau}\left(\mathbb{R}^{d}\right)
$$

which directly extends the well-known classical case from $\tau=0$ to $\tau \in[0, \infty)$, $p \in(0, \infty), q \in(0, \infty]$ and $s \in \mathbb{R}$. Moreover, it is known from [45, Proposition 2.6] that

$$
A_{p, q}^{s, \tau}\left(\mathbb{R}^{d}\right) \hookrightarrow B_{\infty, \infty}^{s+d\left(\tau-\frac{1}{p}\right)}\left(\mathbb{R}^{d}\right) .
$$

The following remarkable feature was proved in [42].

Proposition 2.7 Let $s \in \mathbb{R}, \tau \in[0, \infty)$ and $p, q \in(0, \infty]$ (with $p<\infty$ in the $F$-case). If either $\tau>\frac{1}{p}$ or $\tau=\frac{1}{p}$ and $q=\infty$, then $A_{p, q}^{s, \tau}\left(\mathbb{R}^{d}\right)=B_{\infty, \infty}^{s+d\left(\tau-\frac{1}{p}\right)}\left(\mathbb{R}^{d}\right)$.

As for the scale $\mathcal{A}_{u, p, q}^{s}\left(\mathbb{R}^{d}\right)$ the counterparts to (2.8)-(2.10) read as

$$
\mathcal{A}_{u, p, r}^{s+\varepsilon}\left(\mathbb{R}^{d}\right) \hookrightarrow \mathcal{A}_{u, p, q}^{s}\left(\mathbb{R}^{d}\right) \quad \text { if } \quad \varepsilon>0, \quad r \in(0, \infty],
$$


and

$$
\mathcal{A}_{u, p, q_{1}}^{s}\left(\mathbb{R}^{d}\right) \hookrightarrow \mathcal{A}_{u, p, q_{2}}^{s}\left(\mathbb{R}^{d}\right) \quad \text { if } \quad q_{1} \leq q_{2} .
$$

However, there also exist some differences. Sawano proved in [27] that, for $s \in \mathbb{R}$ and $0<p<u<\infty$,

$$
\mathcal{N}_{u, p, \min \{p, q\}}^{s}\left(\mathbb{R}^{d}\right) \hookrightarrow \mathcal{E}_{u, p, q}^{s}\left(\mathbb{R}^{d}\right) \hookrightarrow \mathcal{N}_{u, p, \infty}^{s}\left(\mathbb{R}^{d}\right)
$$

where, for the latter embedding, $r=\infty$ cannot be improved-unlike in case of $u=p$ [see (2.10) with $\tau=0$ ]. More precisely,

$\mathcal{E}_{u, p, q}^{s}\left(\mathbb{R}^{d}\right) \hookrightarrow \mathcal{N}_{u, p, r}^{s}\left(\mathbb{R}^{d}\right)$ if, and only if, $r=\infty$ or $u=p$ and $r \geq \max \{p, q\}$.

On the other hand, Mazzucato has shown in [21, Proposition 4.1] that

$$
\mathcal{E}_{u, p, 2}^{0}\left(\mathbb{R}^{d}\right)=\mathcal{M}_{u, p}\left(\mathbb{R}^{d}\right), \quad 1<p \leq u<\infty,
$$

in particular,

$$
\mathcal{E}_{p, p, 2}^{0}\left(\mathbb{R}^{d}\right)=L_{p}\left(\mathbb{R}^{d}\right)=F_{p, 2}^{0}\left(\mathbb{R}^{d}\right), \quad p \in(1, \infty)
$$

Remark 2.8 We obtained a lot more embedding results within the scales of spaces $\mathcal{A}_{u, p, q}^{s}\left(\mathbb{R}^{d}\right)$ and $A_{p, q}^{s, \tau}\left(\mathbb{R}^{d}\right)$, respectively, in $[14,15,43,44]$, but will recall some of them in detail below as far as needed for our argument. We turn to the relation between the two scales of smoothness Morrey spaces. Let $s, u, p$ and $q$ be as in Definition 2.4 and $\tau \in[0, \infty)$. It is known from [45, Corollary 3.3, p. 64] that

$$
\mathcal{N}_{u, p, q}^{s}\left(\mathbb{R}^{d}\right) \hookrightarrow B_{p, q}^{s, \tau}\left(\mathbb{R}^{d}\right) \quad \text { with } \quad \tau=\frac{1}{p}-\frac{1}{u} .
$$

Moreover, the above embedding is proper if $\tau>0$ and $q<\infty$. If $\tau=0$ or $q=\infty$, then both spaces coincide with each other, in particular,

$$
\mathcal{N}_{u, p, \infty}^{s}\left(\mathbb{R}^{d}\right)=B_{p, \infty}^{s, \frac{1}{p}-\frac{1}{u}}\left(\mathbb{R}^{d}\right)
$$

As for the $F$-spaces, if $0 \leq \tau<1 / p$, then

$$
F_{p, q}^{s, \tau}\left(\mathbb{R}^{d}\right)=\mathcal{E}_{u, p, q}^{s}\left(\mathbb{R}^{d}\right) \quad \text { with } \quad \tau=\frac{1}{p}-\frac{1}{u}, \quad 0<p \leq u<\infty
$$

cf. [45, Corollary 3.3, p. 63]. Moreover, if $p \in(0, \infty)$ and $q \in(0, \infty]$, then

$$
F_{p, q}^{s, \frac{1}{p}}\left(\mathbb{R}^{d}\right)=F_{\infty, q}^{s}\left(\mathbb{R}^{d}\right)=B_{q, q}^{s, \frac{1}{q}}\left(\mathbb{R}^{d}\right)
$$

cf. [32, Propositions 3.4 and 3.5] and [33, Remark 10]. 
For later use we recall the definition of the space bmo $\left(\mathbb{R}^{d}\right)$, i.e., the local (nonhomogeneous) space of functions of bounded mean oscillation, consisting of all locally integrable functions $f \in L_{1}^{\text {loc }}\left(\mathbb{R}^{d}\right)$ satisfying that

$$
\left\|f\left|\operatorname{bmo}\left(\mathbb{R}^{d}\right) \|:=\sup _{|Q| \leq 1} \frac{1}{|Q|} \int_{Q}\right| f(x)-f_{Q}\left|\mathrm{~d} x+\sup _{|Q|>1} \frac{1}{|Q|} \int_{Q}\right| f(x) \mid \mathrm{d} x<\infty,\right.
$$

where $Q$ appearing in the above definition runs over all cubes in $\mathbb{R}^{d}$, and $f_{Q}$ denotes the mean value of $f$ with respect to $Q$, namely, $f_{Q}:=\frac{1}{|Q|} \int_{Q} f(x) \mathrm{d} x$, cf. [35, 2.2.2(viii)]. Hence the above result (2.19) implies, in particular,

$$
\operatorname{bmo}\left(\mathbb{R}^{d}\right)=F_{\infty, 2}^{0}\left(\mathbb{R}^{d}\right)=F_{p, 2}^{0,1 / p}\left(\mathbb{R}^{d}\right), \quad 0<p<\infty
$$

Remark 2.9 In contrast to this approach, Triebel followed the original MorreyCampanato ideas to develop local spaces $\mathcal{L}^{r} A_{p, q}^{s}\left(\mathbb{R}^{d}\right)$ in [38], and so-called 'hybrid' spaces $L^{r} A_{p, q}^{s}\left(\mathbb{R}^{d}\right)$ in [39], where $0<p<\infty, 0<q \leq \infty, s \in \mathbb{R}$, and $-\frac{d}{p} \leq r<\infty$. This construction is based on wavelet decompositions and also combines local and global elements as in Definitions 2.1 and 2.4. However, Triebel proved in [39, Chapter 3] that

$$
L^{r} A_{p, q}^{s}\left(\mathbb{R}^{d}\right)=A_{p, q}^{s, \tau}\left(\mathbb{R}^{d}\right), \quad \tau=\frac{1}{p}+\frac{r}{d}
$$

in all admitted cases. Therefore we do not have to deal with these spaces separately in the sequel.

\subsection{Spaces on domains}

We assume that $\Omega$ is a bounded domain in $\mathbb{R}^{d}$. We consider smoothness Morrey spaces on $\Omega$ defined by restriction. Let $\mathcal{D}(\Omega)$ be the set of all infinitely differentiable functions supported in $\Omega$ and denote by $\mathcal{D}^{\prime}(\Omega)$ its dual. If $\Omega$ is a $C^{\infty}$ domain, then we are able to define the extension operator ext : $\mathcal{D}(\Omega) \rightarrow \mathcal{S}\left(\mathbb{R}^{d}\right)$, cf. [31], the restriction operator re $: \mathcal{S}^{\prime}\left(\mathbb{R}^{d}\right) \rightarrow \mathcal{D}^{\prime}(\Omega)$ can be defined naturally as an adjoint operator

$$
\langle\operatorname{re}(f), \varphi\rangle=\langle f, \operatorname{ext}(\varphi)\rangle, \quad f \in \mathcal{S}^{\prime}\left(\mathbb{R}^{d}\right),
$$

where $\varphi \in \mathcal{D}(\Omega)$. We will write $\left.f\right|_{\Omega}=\operatorname{re}(f)$.

Definition 2.10 Let $s \in \mathbb{R}$ and $q \in(0, \infty]$.

(i) Let $0<p \leq u<\infty$ or $p=u=\infty$ (with $u<\infty$ in case of $\mathcal{A}=\mathcal{E}$ ). Then $\mathcal{A}_{u, p, q}^{s}(\Omega)$ is defined by

$$
\mathcal{A}_{u, p, q}^{s}(\Omega):=\left\{f \in \mathcal{D}^{\prime}(\Omega): f=\left.g\right|_{\Omega} \text { for some } g \in \mathcal{A}_{u, p, q}^{s}\left(\mathbb{R}^{d}\right)\right\}
$$


endowed with the quasi-norm

$$
\left\|f \mid \mathcal{A}_{u, p, q}^{s}(\Omega)\right\|:=\inf \left\{\left\|g\left|\mathcal{A}_{u, p, q}^{s}\left(\mathbb{R}^{d}\right) \|: f=g\right|_{\Omega}, g \in \mathcal{A}_{u, p, q}^{s}\left(\mathbb{R}^{d}\right)\right\} .\right.
$$

(ii) Let $\tau \in[0, \infty)$ and $p \in(0, \infty]$ (with $p<\infty$ in case of $A=F)$. Then $A_{p, q}^{s, \tau}(\Omega)$ is defined by

$$
A_{p, q}^{s, \tau}(\Omega):=\left\{f \in \mathcal{D}^{\prime}(\Omega): f=\left.g\right|_{\Omega} \text { for some } g \in A_{p, q}^{s, \tau}\left(\mathbb{R}^{d}\right)\right\}
$$

endowed with the quasi-norm

$$
\left\|f \mid A_{p, q}^{s, \tau}(\Omega)\right\|:=\inf \left\{\left\|g\left|A_{p, q}^{s, \tau}\left(\mathbb{R}^{d}\right) \|: f=g\right|_{\Omega}, g \in A_{p, q}^{s, \tau}\left(\mathbb{R}^{d}\right)\right\} .\right.
$$

Remark 2.11 The spaces $\mathcal{A}_{u, p, q}^{s}(\Omega)$ and $A_{p, q}^{s, \tau}(\Omega)$ are quasi-Banach spaces (Banach spaces for $p, q \geq 1$ ). When $u=p$ or $\tau=0$ we re-obtain the usual Besov and Triebel-Lizorkin spaces defined on bounded domains. Several properties of the spaces $\mathcal{A}_{u, p, q}^{s}(\Omega)$, including the extension property, were studied in [31]. As for the spaces $A_{p, q}^{s, \tau}(\Omega)$ we also refer to [45, Section 6.4.2]. In particular, if the domain is smooth then, according to [45, Theorem 6.13], there exists a linear and bounded extension operator

$$
\operatorname{ext}_{\tau}: A_{p, q}^{s, \tau}(\Omega) \rightarrow A_{p, q}^{s, \tau}\left(\mathbb{R}^{d}\right), \quad \text { where } 1 \leq p<\infty, 0<q \leq \infty, s \in \mathbb{R}, \tau \geq 0,
$$

such that

$$
\text { re } \circ \operatorname{ext}_{\tau}=\text { id in } A_{p, q}^{s, \tau}(\Omega) \text {, }
$$

where re: $A_{p, q}^{s, \tau}\left(\mathbb{R}^{d}\right) \rightarrow A_{p, q}^{s, \tau}(\Omega)$ is the restriction operator as above.

Several types of embeddings related to these scales were already considered for bounded smooth domains. For instance, embeddings within the scale of spaces $\mathcal{A}_{u, p, q}^{s}(\Omega)$ as well as to classical spaces like $C(\Omega)$ or $L_{r}(\Omega)$ were investigated in $[14,15]$. In [12] we studied the question under what assumptions these spaces consist of regular distributions only. Moreover, in [43] we considered the approximation numbers of some special compact embedding of $A_{p, q}^{s, \tau}(\Omega)$ into $L_{\infty}(\Omega)$.

Remark 2.12 Let us mention that we have the counterparts of many continuous embeddings stated in the previous subsection for spaces on $\mathbb{R}^{d}$ when dealing with spaces restricted to bounded domains. This concerns, in particular, the elementary embeddings and coincidences (2.8)-(2.10), Proposition 2.7 and (2.12)-(2.18).

For a matter of completion, we finish this subsection by giving the definition of $\operatorname{bmo}(\Omega)$, that we will use later on. As previously, this is done by restriction, that is, $\operatorname{bmo}(\Omega)$ is defined as being the space of all restrictions to $\Omega$ of functions in $\operatorname{bmo}\left(\mathbb{R}^{d}\right)$, equipped with the norm

$$
\|f \mid \operatorname{bmo}(\Omega)\|:=\inf \left\{\left\|g\left|\operatorname{bmo}\left(\mathbb{R}^{d}\right) \|: f=g\right|_{\Omega}, g \in \operatorname{bmo}\left(\mathbb{R}^{d}\right)\right\} .\right.
$$




\subsection{Entropy numbers}

As explained in the beginning already, our main concern in this paper is to characterize the compactness of embeddings in further detail. Therefore we briefly recall the concepts of entropy and approximation numbers.

Definition 2.13 Let $X$ and $Y$ be two complex (quasi-) Banach spaces, $k \in \mathbb{N}$ and let $T \in \mathcal{L}(X, Y)$ be a linear and continuous operator from $X$ into $Y$.

(i) The kth entropy number $e_{k}(T)$ of $T$ is the infimum of all numbers $\varepsilon>0$ such that there exist $2^{k-1}$ balls in $Y$ of radius $\varepsilon$ which cover the image $T B_{X}$ of the unit ball $B_{X}=\{x \in X:\|x \mid X\| \leq 1\}$.

(ii) The kth approximation number $a_{k}(T)$ of $T$ is defined by

$$
a_{k}(T)=\inf \{\|T-S\|: S \in \mathcal{L}(X, Y), \operatorname{rank} S<k\}, \quad k \in \mathbb{N} .
$$

Remark 2.14 For details and properties of entropy and approximation numbers we refer to [3,4,18,25] (restricted to the case of Banach spaces), and [7] for some extensions to quasi-Banach spaces. Among other features we only want to mention the multiplicativity of entropy numbers: let $X, Y, Z$ be complex (quasi-) Banach spaces and $T_{1} \in \mathcal{L}(X, Y), T_{2} \in \mathcal{L}(Y, Z)$. Then

$$
e_{k_{1}+k_{2}-1}\left(T_{2} \circ T_{1}\right) \leq e_{k_{1}}\left(T_{1}\right) e_{k_{2}}\left(T_{2}\right), \quad k_{1}, k_{2} \in \mathbb{N}
$$

Note that one has in general $\lim _{k \rightarrow \infty} e_{k}(T)=0$ if, and only if, $T$ is compact. The last equivalence justifies the saying that entropy numbers measure 'how compact' an operator acts. This is one reason to study the asymptotic behavior of entropy numbers (that is, their decay) for compact operators in detail.

Approximation numbers share many of the basic features of entropy numbers, but are different in some respect. They can-unlike entropy numbers-be regarded as special s-numbers, a concept introduced by Pietsch [24, Section 11]. Of special importance is the close connection of both concepts, entropy numbers as well as approximation numbers, with spectral theory, in particular, the estimate of eigenvalues. We refer to the monographs $[3,4,7,18,25]$ for further details.

Remark 2.15 We recall what is well-known in the case of the embedding

$$
\operatorname{id}_{A}: A_{p_{1}, q_{1}}^{s_{1}}(\Omega) \rightarrow A_{p_{2}, q_{2}}^{s_{2}}(\Omega)
$$

where $-\infty<s_{2} \leq s_{1}<\infty, 0<p_{1}, p_{2} \leq \infty\left(p_{1}, p_{2}<\infty\right.$ in the $F$-case $)$, $0<q_{1}, q_{2} \leq \infty$, and the spaces $A_{p, q}^{s}(\Omega)$ are defined by restriction. Let

$$
\delta=s_{1}-s_{2}-d\left(\frac{1}{p_{1}}-\frac{1}{p_{2}}\right), \quad \delta_{+}=s_{1}-s_{2}-d\left(\frac{1}{p_{1}}-\frac{1}{p_{2}}\right)_{+} .
$$


Then $\mathrm{id}_{A}$ is compact when $\delta_{+}>0$; cf. [7, (2.5.1/10)]. In this situation Edmunds and Triebel proved in $[5,6]$ (see also [7, Theorem 3.3.3/2]) that

$$
e_{k}\left(\operatorname{id}_{A}\right) \sim k^{-\frac{s_{1}-s_{2}}{d}}, \quad k \in \mathbb{N},
$$

where $s_{1} \geq s_{2}, 0<p_{1}, p_{2} \leq \infty\left(p_{1}, p_{2}<\infty\right.$ in the $F$-case $), 0<q_{1}, q_{2} \leq \infty$, and $\delta_{+}>0$. It was originally proved there for smooth domains, but the extension to arbitrary bounded domains is also covered by [37, Theorem 1.92]. In the case of approximation numbers the situation is more complicated; the result of Edmunds and Triebel for smooth domains in [7, Theorem 3.3.4], partly improved by Caetano [2], reads as

$$
a_{k}\left(\operatorname{id}_{A}\right) \sim k^{-\frac{\delta_{+}}{d}-\varkappa}, \quad k \in \mathbb{N},
$$

with

$$
\varkappa=\left(\frac{\min \left\{p_{1}^{\prime}, p_{2}\right\}}{2}-1\right)_{+} \cdot \min \left\{\frac{\delta}{d}, \frac{1}{\min \left\{p_{1}^{\prime}, p_{2}\right\}}\right\},
$$

where $\delta$ is given by (2.26) and $p_{1}^{\prime}$ denotes the conjugate of $p_{1}$ defined by $\frac{1}{p_{1}}+\frac{1}{p_{1}^{\prime}}=1$ if $1 \leq p_{1} \leq \infty$ and $p_{1}^{\prime}=\infty$ if $0<p_{1}<1$. The above asymptotic result is almost complete now, apart from the restrictions that $\left(p_{1}, p_{2}\right) \neq(1, \infty)$ or $\frac{\delta}{d} \neq \frac{1}{\min \left\{p_{1}^{\prime}, p_{2}\right\}}$ when $0<p_{1}<2<p_{2} \leq \infty$. Note that $\varkappa=0$ unless $p_{1}<2<p_{2}$, and $\delta \geq \delta_{+}$ with $\delta=\delta_{+}$if $p_{1} \leq p_{2}$.

\section{Compact embeddings}

First we recall our compactness result as obtained in [14] (for $\mathcal{A}=\mathcal{N}$ ) and [15] (for $\mathcal{A}=\mathcal{E}$ ), with a supplement related to arbitrary bounded domains proved in [17]. We shall heavily rely on this result in our argument below.

Convention Here and in the sequel we shall understand $\frac{p_{i}}{u_{i}}=1$ in case of $p_{i}=u_{i}=\infty$, $i=1,2$.

Theorem 3.1 Let $s_{i} \in \mathbb{R}, 0<q_{i} \leq \infty, 0<p_{i} \leq u_{i}<\infty$, or, in the case of $\mathcal{N}$-spaces, allow also $p_{i}=u_{i}=\infty, i=1,2$. Then the embedding

$$
\operatorname{id}_{\mathcal{A}}: \mathcal{A}_{u_{1}, p_{1}, q_{1}}^{s_{1}}(\Omega) \hookrightarrow \mathcal{A}_{u_{2}, p_{2}, q_{2}}^{s_{2}}(\Omega)
$$

is compact if, and only if, the following condition holds:

$$
\frac{s_{1}-s_{2}}{d}>\max \left\{0, \frac{1}{u_{1}}-\frac{1}{u_{2}}, \frac{p_{1}}{u_{1}}\left(\frac{1}{p_{1}}-\frac{1}{p_{2}}\right)\right\} .
$$

In particular, if $p_{1}=u_{1}=\infty$ and $\mathcal{A}_{u_{1}, p_{1}, q_{1}}^{s_{1}}=\mathcal{N}_{u_{1}, p_{1}, q_{1}}^{s_{1}}$, then $\mathrm{id}_{\mathcal{A}}$ given by (3.1) is compact if, and only if, $s_{1}>s_{2}$. If $p_{2}=u_{2}=\infty$ and $\mathcal{A}_{u_{2}, p_{2}, q_{2}}^{s_{2}}=\mathcal{N}_{u_{2}, p_{2}, q_{2}}^{s_{2}}$, then $\operatorname{id}_{\mathcal{A}}$ is compact if, and only if, $\frac{s_{1}-s_{2}}{d}>\frac{1}{u_{1}}$. 
Proof The cases when $0<p_{i} \leq u_{i}<\infty, i=1,2$, were proved in [14,15] for $\mathcal{A}=\mathcal{N}$ and $\mathcal{A}=\mathcal{E}$ respectively. So we are left with the cases $p_{1}=u_{1}=\infty$ or $p_{2}=u_{2}=\infty$.

At first, let us consider the case when $p_{1}=u_{1}=\infty$ and $\mathcal{A}_{u_{1}, p_{1}, q_{1}}^{s_{1}}=\mathcal{N}_{u_{1}, p_{1}, q_{1}}^{s_{1}}$. If $s_{1}-s_{2}>0$, the compactness of $\operatorname{id}_{\mathcal{A}}$ follows from

$$
\mathcal{N}_{\infty, \infty, q_{1}}^{s_{1}}(\Omega)=B_{\infty, q_{1}}^{s_{1}}(\Omega) \hookrightarrow A_{u_{2}, q_{2}}^{s_{2}}(\Omega) \hookrightarrow \mathcal{A}_{u_{2}, p_{2}, q_{2}}^{s_{2}}(\Omega),
$$

as the first embedding is compact when $s_{1}-s_{2}>0$.

Now we assume that $\mathrm{id}_{\mathcal{A}}$ is compact. We have

$$
B_{\infty, q_{1}}^{s_{1}}(\Omega)=\mathcal{N}_{\infty, \infty, q_{1}}^{s_{1}}(\Omega) \hookrightarrow \mathcal{A}_{u_{2}, p_{2}, q_{2}}^{s_{2}}(\Omega) \hookrightarrow A_{p_{2}, q_{2}}^{s_{2}}(\Omega),
$$

where the last embedding was proved in $[14,15]$. Then, the compactness of the first embedding implies the compactness of the embedding between the outer spaces, which in turn implies $s_{1}-s_{2}>0$.

Now let $p_{2}=u_{2}=\infty, \mathcal{A}_{u_{2}, p_{2}, q_{2}}^{s_{2}}=\mathcal{N}_{u_{2}, p_{2}, q_{2}}^{s_{2}}$ and $\frac{s_{1}-s_{2}}{d}>\frac{1}{u_{1}}$. As the case $p_{1}=u_{1}=\infty$ (when $\left.\mathcal{A}=\mathcal{N}\right)$ is already covered by our preceding observation, we may further assume that $0<p_{1} \leq u_{1}<\infty$. By a straightforward extension of our continuity result in [14, Theorem 3.1] (to the cases when $p_{i}=u_{i}=\infty$ for $i=1$ or $i=2$ ) we have the continuous embedding

$$
\mathcal{N}_{u_{1}, p_{1}, q_{1}}^{s_{1}}(\Omega) \hookrightarrow B_{\infty, \infty}^{s_{1}-\frac{d}{u_{1}}}(\Omega) .
$$

Moreover, in case of $s_{1}-\frac{d}{u_{1}}>s_{2}$, it is well-known that the embedding

$$
B_{\infty, \infty}^{s_{1}-\frac{d}{u_{1}}}(\Omega) \hookrightarrow B_{\infty, q_{2}}^{s_{2}}(\Omega)=\mathcal{N}_{\infty, \infty, q_{2}}^{s_{2}}(\Omega)
$$

is compact. Thus $\mathcal{N}_{u_{1}, p_{1}, q_{1}}^{s_{1}}(\Omega) \hookrightarrow \mathcal{N}_{\infty, \infty, q_{2}}^{s_{2}}(\Omega)$ compactly for any $q_{1}, q_{2} \in(0, \infty]$. The compactness of $\mathcal{E}_{u_{1}, p_{1}, q_{1}}^{s_{1}}(\Omega) \hookrightarrow \mathcal{N}_{\infty, \infty, q_{2}}^{s_{2}}(\Omega)$ is then a consequence of (2.14).

The necessity follows from the following chain of embeddings

$$
B_{u_{1}, q_{1}}^{s_{1}}(\Omega) \hookrightarrow \mathcal{N}_{u_{1}, p_{1}, q_{1}}^{s_{1}}(\Omega) \hookrightarrow \mathcal{N}_{\infty, \infty, q_{2}}^{s_{2}}(\Omega)=B_{\infty, q_{2}}^{s_{2}}(\Omega)
$$

in the same way as above. Finally we apply (2.14) for the case $\mathcal{A}_{u_{1}, p_{1}, q_{1}}^{s_{1}}=\mathcal{E}_{u_{1}, p_{1}, q_{1}}^{s_{1}}$.

Now we give the counterpart of Theorem 3.1 for Besov-type and Triebel-Lizorkintype spaces. For convenience we use some abbreviation for the following expression, which plays an essential role in the sequel. So let us denote

$$
\gamma\left(\tau_{1}, \tau_{2}, p_{1}, p_{2}\right):=\max \left\{\left(\tau_{2}-\frac{1}{p_{2}}\right)_{+}-\left(\tau_{1}-\frac{1}{p_{1}}\right)_{+}, \frac{1}{p_{1}}-\tau_{1}-\min \left\{\frac{1}{p_{2}}-\tau_{2}, \frac{1}{p_{2}}\left(1-p_{1} \tau_{1}\right)_{+}\right\}\right\} .
$$


Theorem 3.2 Let $s_{i} \in \mathbb{R}, 0<q_{i} \leq \infty, 0<p_{i} \leq \infty$ (with $p_{i}<\infty$ in case of $\left.A=F\right)$, $\tau_{i} \geq 0, i=1,2$. The embedding

$$
\operatorname{id}_{\tau}: A_{p_{1}, q_{1}}^{s_{1}, \tau_{1}}(\Omega) \hookrightarrow A_{p_{2}, q_{2}}^{s_{2}, \tau_{2}}(\Omega)
$$

is compact if, and only if, the following condition holds:

$$
\frac{s_{1}-s_{2}}{d}>\gamma\left(\tau_{1}, \tau_{2}, p_{1}, p_{2}\right)
$$

Proof We shall use sharp embeddings and identities like (2.11), Proposition 2.7 and (2.18) (all adapted to spaces restricted to the domain $\Omega$, recall Remark 2.12) together with our previous result Theorem 3.1 several times. Therefore we shall always distinguish below between the cases $\tau_{i}<\frac{1}{p_{i}}$ and $\tau_{i} \geq \frac{1}{p_{i}}$ (with some additional restrictions on $q_{i}$ occasionally), $i=1,2$. For that reason it seems convenient to reformulate condition (3.10) according to these cases; that is, the goal is to prove that $\mathrm{id}_{\tau}$ given by (3.9) is compact if, and only if,

$$
\frac{s_{1}-s_{2}}{d}> \begin{cases}\frac{1}{p_{1}}-\tau_{1}-\frac{1}{p_{2}}+\tau_{2}, & \text { if } \tau_{2} \geq \frac{1}{p_{2}}, \\ \frac{1}{p_{1}}-\tau_{1}, & \text { if } \tau_{1} \geq \frac{1}{p_{1}}, \tau_{2}<\frac{1}{p_{2}}, \\ \max \left\{0, \frac{1}{p_{1}}-\tau_{1}-\frac{1}{p_{2}}+\max \left\{\tau_{2}, \frac{p_{1}}{p_{2}} \tau_{1}\right\}\right\}, & \text { if } \tau_{1}<\frac{1}{p_{1}}, \tau_{2}<\frac{1}{p_{2}} .\end{cases}
$$

Step 1. Let us assume that $\tau_{2} \geq \frac{1}{p_{2}}$ with $q_{2}=\infty$ if $\tau_{2}=\frac{1}{p_{2}}$. First we prove the sufficiency of (3.11) for the compactness of $\mathrm{id}_{\tau}$, that is, we assume now

$$
s_{1}+d\left(\tau_{1}-\frac{1}{p_{1}}\right)>s_{2}+d\left(\tau_{2}-\frac{1}{p_{2}}\right) .
$$

Then the embedding (2.11) and Proposition 2.7 yield

$$
A_{p_{1}, q_{1}}^{s_{1}, \tau_{1}}(\Omega) \hookrightarrow B_{\infty, \infty}^{s_{1}+d\left(\tau_{1}-\frac{1}{p_{1}}\right)}(\Omega) \hookrightarrow B_{\infty, \infty}^{s_{2}+d\left(\tau_{2}-\frac{1}{p_{2}}\right)}(\Omega)=A_{p_{2}, q_{2}}^{s_{2}, \tau_{2}}(\Omega)
$$

and the embedding between the Besov spaces is compact. So $\mathrm{id}_{\tau}$ is compact.

Now we turn to the necessity of (3.12) for the compactness of $\operatorname{id}_{\tau}$. So we assume that $\mathrm{id}_{\tau}$ given by (3.9) is compact. Let first $\tau_{1} \geq \frac{1}{p_{1}}$ with $q_{1}=\infty$ if $\tau_{1}=\frac{1}{p_{1}}$. Then by Proposition 2.7 we obtain

$$
B_{\infty, \infty}^{s_{1}+d\left(\tau_{1}-\frac{1}{p_{1}}\right)}(\Omega)=A_{p_{1}, q_{1}}^{s_{1}, \tau_{1}}(\Omega) \hookrightarrow A_{p_{2}, q_{2}}^{s_{2}, \tau_{2}}(\Omega)=B_{\infty, \infty}^{s_{2}+d\left(\tau_{2}-\frac{1}{p_{2}}\right)}(\Omega)
$$

which results in a compact embedding between the outer Besov spaces. This is wellknown to imply (3.12) as desired. A similar argument works for $p_{1}=\infty, \tau_{1}=0$ and 
$q_{1}<\infty$, since then we have

$$
B_{\infty, q_{1}}^{s_{1}}(\Omega)=B_{p_{1}, q_{1}}^{s_{1}, \tau_{1}}(\Omega) \hookrightarrow A_{p_{2}, q_{2}}^{s_{2}, \tau_{2}}(\Omega)=B_{\infty, \infty}^{s_{2}+d\left(\tau_{2}-\frac{1}{p_{2}}\right)}(\Omega) .
$$

Let now $0<\tau_{1} \leq \frac{1}{p_{1}}$ with $q_{1}<\infty$ if $\tau_{1}=\frac{1}{p_{1}}$. We take $p_{0} \in(0, \infty)$ such that $\frac{1}{p_{0}}>\frac{1}{p_{1}}-\tau_{1}$ and $q_{0}=\min \left\{p_{1}, q_{1}\right\}$. Then Corollary 5.2 in [44] implies

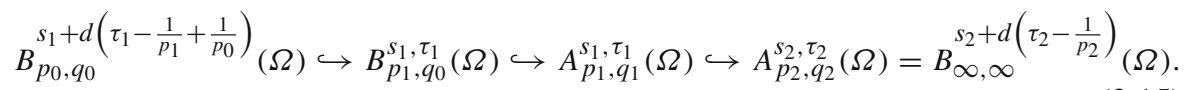

Once more the compactness of $\mathrm{id}_{\tau}$ implies (3.12). If $\tau_{1}=0$, then $B_{p_{1}, q_{0}}^{s_{1}}(\Omega)$ can be embedded into $A_{p_{1}, q_{1}}^{s_{1}, \tau_{1}}(\Omega)$ and the similar argument holds. This concludes the proof in that case of $\tau_{2} \geq \frac{1}{p_{2}}$ with $q_{2}=\infty$ if $\tau_{2}=\frac{1}{p_{2}}$.

Step 2 Next we assume that $0 \leq \tau_{2}<\frac{1}{p_{2}}$ and benefit from the coincidence (2.18).

Substep 2.1 If also $0 \leq \tau_{1}<\frac{1}{p_{1}}$, then (3.10) reads as

$$
\frac{s_{1}-s_{2}}{d}>\max \left\{0, \frac{1}{p_{1}}-\tau_{1}-\frac{1}{p_{2}}+\max \left\{\tau_{2}, \frac{p_{1}}{p_{2}} \tau_{1}\right\}\right\} .
$$

For the $F$-spaces, the result immediately follows from coincidence (2.18) and Theorem 3.1. Note that in this case (3.2) coincides with the last line in (3.11) in view of $\frac{1}{u_{i}}=$ $\frac{1}{p_{i}}-\tau_{i}, i=1,2$, as required by (2.18).

We shall then prove the result for the Besov scale. At first, let us assume (3.16) holds. Then (2.9), (2.16) and (2.17) yield

$$
B_{p_{1}, q_{1}}^{s_{1}, \tau_{1}}(\Omega) \hookrightarrow B_{p_{1}, \infty}^{s_{1}, \tau_{1}}(\Omega)=\mathcal{N}_{u_{1}, p_{1}, \infty}^{s_{1}}(\Omega) \hookrightarrow \mathcal{N}_{u_{2}, p_{2}, q_{2}}^{s_{2}}(\Omega) \hookrightarrow B_{p_{2}, q_{2}}^{s_{2}, \tau_{2}}(\Omega)
$$

and the embedding between the Besov-Morrey spaces is compact. Therefore $\mathrm{id}_{\tau}$ is compact.

We now turn to the necessity and assume that $\mathrm{id}_{\tau}$ is compact. Then

$$
\mathcal{N}_{u_{1}, p_{1}, q_{1}}^{s_{1}}(\Omega) \hookrightarrow B_{p_{1}, q_{1}}^{s_{1}, \tau_{1}}(\Omega) \hookrightarrow B_{p_{2}, q_{2}}^{s_{2}, \tau_{2}}(\Omega) \hookrightarrow B_{p_{2}, \infty}^{s_{2}, \tau_{2}}(\Omega)=\mathcal{N}_{u_{2}, p_{2}, \infty}^{s_{2}}(\Omega),
$$

where we have used again (2.16), (2.9) and the coincidence (2.17). In view of Theorem 3.1 this leads to the desired condition (3.16).

Substep 2.2 An analogous argument works for $\tau_{1} \geq \frac{1}{p_{1}}$ with $q_{1}=\infty$ if $\tau_{1}=\frac{1}{p_{1}}$, cf. Proposition 2.7. This time (3.10) coincides with the second line of (3.11). So for the sufficiency we assume that

$$
\frac{s_{1}-s_{2}}{d}>\frac{1}{p_{1}}-\tau_{1}
$$


Then Proposition 2.7, the embedding (2.16) and the coincidence (2.18) give

$$
A_{p_{1}, q_{1}}^{s_{1}, \tau_{1}}(\Omega)=B_{\infty, \infty}^{s_{1}+d\left(\tau_{1}-\frac{1}{p_{1}}\right)}(\Omega)=\mathcal{N}_{\infty, \infty, \infty}^{s_{1}+d\left(\tau_{1}-\frac{1}{p_{1}}\right)}(\Omega) \hookrightarrow \mathcal{A}_{u_{2}, p_{2}, q_{2}}^{s_{2}}(\Omega) \hookrightarrow A_{p_{2}, q_{2}}^{s_{2}, \tau_{2}}(\Omega) .
$$

Then, by Theorem $3.1, \mathrm{id}_{\tau}$ is compact. Conversely, let us assume that $\mathrm{id}_{\tau}$ is compact. We benefit from Proposition 2.7 and the coincidences (2.17) and (2.18) to obtain

$$
\mathcal{N}_{\infty, \infty, \infty}^{s_{1}+d\left(\tau_{1}-\frac{1}{p_{1}}\right)}(\Omega)=B_{\infty, \infty}^{s_{1}+d\left(\tau_{1}-\frac{1}{p_{1}}\right)}(\Omega)=A_{p_{1}, q_{1}}^{s_{1}, \tau_{1}}(\Omega) \hookrightarrow A_{p_{2}, q_{2}}^{s_{2}, \tau_{2}}(\Omega) \hookrightarrow \mathcal{N}_{u_{2}, p_{2}, \infty}^{s_{2}}(\Omega) .
$$

In case of $A=F$, the last embedding is true due to the elementary embeddings (2.14). Therefore, the compactness of $\mathrm{id}_{\tau}$ and Theorem 3.1 lead to the desired condition $\frac{s_{1}-s_{2}}{d}>\frac{1}{p_{1}}-\tau_{1}$.

Substep 2.3 Assume finally $\tau_{1}=\frac{1}{p_{1}}$ with $q_{1}<\infty$. Note that in this case, due to the middle line of (3.11), the condition (3.10) reads as $s_{1}>s_{2}$. We apply (2.11) to obtain

$$
\begin{aligned}
& A_{p_{1}, q_{1}}^{s_{1}, \tau_{1}}(\Omega)=A_{p_{1}, q_{1}}^{s_{1}, \frac{1}{p_{1}}}(\Omega) \hookrightarrow B_{\infty, \infty}^{s_{1}}(\Omega) \hookrightarrow \mathcal{A}_{u_{2}, p_{2}, q_{2}}^{s_{2}}(\Omega) \hookrightarrow A_{p_{2}, q_{2}}^{s_{2}, \tau_{2}}(\Omega), \\
& \text { with } \frac{1}{u_{2}}=\frac{1}{p_{2}}-\tau_{2}>0 .
\end{aligned}
$$

Since $B_{\infty, \infty}^{s_{1}}(\Omega)=\mathcal{N}_{\infty, \infty, \infty}^{s_{1}}(\Omega)$, in view of Theorem 3.1 the second embedding is compact for $s_{1}>s_{2}$, and the last embedding is a consequence of (2.16) and (2.18), respectively. Conversely, if $\mathrm{id}_{\tau}$ is compact in this case, then we can argue as follows. Put $\frac{1}{u_{2}}=\frac{1}{p_{2}}-\tau_{2}$. Then [44, Corollary 5.2] (for $A=B$ ) and (2.19) (for $A=F$ ) lead to

$B_{\infty, q_{1}}^{s_{1}}(\Omega) \hookrightarrow A_{p_{1}, q_{1}}^{s_{1}, \tau_{1}}(\Omega) \hookrightarrow A_{p_{2}, q_{2}}^{s_{2}, \tau_{2}}(\Omega) \hookrightarrow A_{p_{2}, v_{2}}^{s_{2}, \tau_{2}}(\Omega)=\mathcal{A}_{u_{2}, p_{2}, v_{2}}^{s_{2}}(\Omega) \hookrightarrow \mathcal{N}_{u_{2}, p_{2}, \infty}^{s_{2}}(\Omega)$

with $v_{2}=\infty$ if $A=B$, and $v_{2} \geq q_{2}$ if $A=F$, where we used (2.17) and (2.18) in the last equality and (2.14) in the last embedding. In view of Theorem 3.1 this leads to $s_{1}>s_{2}$ as required.

Step 3 It remains to deal with $\tau_{2}=\frac{1}{p_{2}}$ and $q_{2}<\infty$. In that case (3.11) always reads as $s_{1}-s_{2}>d\left(\frac{1}{p_{1}}-\tau_{1}\right)$.

Substep 3.1 Assume first $\tau_{1}<\frac{1}{p_{1}}$ and let $\frac{1}{u_{1}}=\frac{1}{p_{1}}-\tau_{1}$. Then by elementary embeddings and the coincidences (2.17) and (2.18),

$$
A_{p_{1}, q_{1}}^{s_{1}, \tau_{1}}(\Omega) \hookrightarrow \mathcal{N}_{u_{1}, p_{1}, \infty}^{s_{1}}(\Omega) \hookrightarrow \mathcal{N}_{\infty, \infty, q_{0}}^{s_{2}}(\Omega)=B_{\infty, q_{0}}^{s_{2}}(\Omega) \hookrightarrow B_{p_{2}, q_{0}}^{s_{2}, \tau_{2}}(\Omega),
$$

and the embedding of the outer spaces is compact for any $q_{0}$, since the second embedding is compact by Theorem 3.1 with (3.10). The last embedding is continuous where 
we apply [44, Theorem 2.5]. If $A=B$, we put $q_{0}=q_{2}$ and the argument is complete, while in case of $A=F$ we choose $q_{0} \leq \min \left\{p_{2}, q_{2}\right\}$ and finally use the continuous embedding into $F_{p_{2}, q_{2}}^{s_{2}, \tau_{2}}(\Omega)$ due to (2.10).

On the other hand, [44, Corollaries 5.2, 5.9] and (2.11) ensure

$$
A_{u_{1}, q_{1}}^{s_{1}}(\Omega) \hookrightarrow A_{p_{1}, q_{1}}^{s_{1}, \tau_{1}}(\Omega) \hookrightarrow A_{p_{2}, q_{2}}^{s_{2}, \tau_{2}}(\Omega) \hookrightarrow B_{\infty, \infty}^{s_{2}}(\Omega),
$$

such that the compactness of $\operatorname{id}_{\tau}$ implies $s_{1}-\frac{d}{u_{1}}>s_{2}$ by the well-known classical results. This proves the necessity of the condition.

Substep 3.2 Assume $\tau_{1} \geq \frac{1}{p_{1}}$ with $q_{1}=\infty$ if $\tau_{1}=\frac{1}{p_{1}}$. We can argue in the same way as above. Let $s_{1}-s_{2}>d\left(\frac{1}{p_{1}}-\tau_{1}\right)$. We choose $s_{0}$ such that $s_{1}-d\left(\frac{1}{p_{1}}-\tau_{1}\right)>s_{0}>s_{2}$. Then by the identities in Proposition 2.7 and (2.19),

$$
A_{p_{1}, q_{1}}^{s_{1}, \tau_{1}}(\Omega)=B_{\infty, \infty}^{s_{1}+d\left(\tau_{1}-\frac{1}{p_{1}}\right)}(\Omega) \hookrightarrow B_{\infty, \infty}^{s_{0}}(\Omega)=F_{p_{2}, \infty}^{s_{0}, \tau_{2}}(\Omega) \hookrightarrow A_{p_{2}, q_{2}}^{s_{2}, \tau_{2}}(\Omega),
$$

where the first embedding is compact for $s_{1}+d\left(\tau_{1}-\frac{1}{p_{1}}\right)>s_{0}$ and the last embedding is continuous for $s_{0}>s_{2}$ by (2.8) and (2.10). Hence id ${ }_{\tau}$ is compact. Conversely, the compactness of $\mathrm{id}_{\tau}$ implies

$$
B_{\infty, \infty}^{s_{1}+d\left(\tau_{1}-\frac{1}{p_{1}}\right)}(\Omega)=A_{p_{1}, q_{1}}^{s_{1}, \tau_{1}}(\Omega) \hookrightarrow A_{p_{2}, q_{2}}^{s_{2}, \tau_{2}}(\Omega) \hookrightarrow B_{\infty, \infty}^{s_{2}}(\Omega)
$$

where we used (2.11) and Proposition 2.7. But the resulting compactness of the outer embedding of Besov spaces leads to the desired condition $s_{1}+d\left(\tau_{1}-\frac{1}{p_{1}}\right)>s_{2}$.

Substep 3.3 Let finally $\tau_{1}=\frac{1}{p_{1}}$ with $q_{1}<\infty$. So we are in the double-limiting case and need to show the compactness of $\mathrm{id}_{\tau}$ if, and only if, $s_{1}>s_{2}$. The sufficiency can be obtained via

$$
A_{p_{1}, q_{1}}^{s_{1}, \tau_{1}}(\Omega) \hookrightarrow B_{\infty, \infty}^{s_{1}}(\Omega) \hookrightarrow B_{\infty, q_{2}}^{s_{2}}(\Omega) \hookrightarrow A_{p_{2}, q_{2}}^{s_{2}, \tau_{2}}(\Omega)
$$

where we use [44, Corollary 5.2] in the last embedding in case of $A=B$, extended by the same argument as above to $A=F$ via (2.10). The second embedding is compact for $s_{1}>s_{2}$.

Conversely, if $\mathrm{id}_{\tau}$ is compact, choose $q_{0} \leq \min \left\{p_{1}, q_{1}\right\}$. Then

$$
B_{\infty, q_{0}}^{s_{1}}(\Omega) \hookrightarrow A_{p_{1}, q_{1}}^{s_{1}, \tau_{1}}(\Omega) \hookrightarrow A_{p_{2}, q_{2}}^{s_{2}, \tau_{2}}(\Omega) \hookrightarrow B_{\infty, \infty}^{s_{2}}(\Omega)
$$

is compact, where we used for the first embedding [44, Corollary 5.2] (with (2.10) for $A=F$ ) again, and (2.11) for the last one. But this implies $s_{1}>s_{2}$.

Remark 3.3 Usually one needs the condition $s_{1}-s_{2}>0$ to prove compactness of this kind of embeddings. Curiously this is not the case when considering spaces of type $B_{p, q}^{s, \tau}$ and $F_{p, q}^{s, \tau}$. This can easily be seen by condition (3.11), for instance when $\tau_{1} \geq \frac{1}{p_{1}}$ and $\tau_{2}<\frac{1}{p_{2}}$. In parallel to (2.11) and Proposition 2.7, this evidences the fact that the parameter $\tau$ modifies indeed the smoothness of these spaces. 
Remark 3.4 We briefly return to our Remark 2.9 which referred to the coincidence of Triebel's hybrid spaces $L^{r} A_{p, q}^{s}$ with the spaces $A_{p, q}^{s, \tau}$ if $\tau=\frac{1}{p}+\frac{r}{d}$. Obviously, defining both by restriction to $\Omega$, this is transferred to spaces on domains. In that sense Theorem 3.2 can be formulated as follows: let $s_{i} \in \mathbb{R}, 0<q_{i} \leq \infty, 0<p_{i}<\infty$, $-\frac{d}{p_{i}} \leq r_{i}<\infty, i=1,2$. Then the embedding

$$
\operatorname{id}_{L}: L^{r_{1}} A_{p_{1}, q_{1}}^{s_{1}}(\Omega) \hookrightarrow L^{r_{2}} A_{p_{2}, q_{2}}^{s_{2}}(\Omega)
$$

is compact if, and only if, the following condition holds:

$$
s_{1}-s_{2}>\max \left\{\left(r_{2}\right)_{+}-\left(r_{1}\right)_{+},-r_{1}+\max \left\{r_{2}, \frac{p_{1}}{p_{2}} \min \left\{r_{1}, 0\right\}\right\}\right\},
$$

i.e.,

$$
s_{1}-s_{2}> \begin{cases}r_{2}-r_{1}, & \text { if } r_{2} \geq 0, \\ -r_{1}, & \text { if } r_{2}<0, r_{1} \geq 0 \\ \max \left\{0,-r_{1}+\max \left\{r_{2}, \frac{p_{1}}{p_{2}} r_{1}\right\}\right\}, & \text { if } r_{2}<0, r_{1}<0 .\end{cases}
$$

We now collect some immediate consequences of the above compactness result. We begin with the case $\tau_{1}=\tau_{2}=\tau \geq 0$.

Corollary 3.5 Let $s_{i} \in \mathbb{R}, 0<q_{i} \leq \infty, 0<p_{i} \leq \infty$ (with $p_{i}<\infty$ in case of $A=F), i=1,2$, and $\tau \geq 0$. The embedding

$$
\operatorname{id}_{\tau}: A_{p_{1}, q_{1}}^{s_{1}, \tau}(\Omega) \hookrightarrow A_{p_{2}, q_{2}}^{s_{2}, \tau}(\Omega)
$$

is compact if, and only if,

$$
\frac{s_{1}-s_{2}}{d}> \begin{cases}\frac{1}{p_{1}}-\frac{1}{p_{2}}, & \text { if } p_{1}<p_{2} \\ \min \left\{0, \frac{1}{p_{1}}-\min \left\{\tau, \frac{1}{p_{2}}\right\}\right\}, & \text { if } p_{1} \geq p_{2}\end{cases}
$$

Proof We apply Theorem 3.2 with $\tau_{1}=\tau_{2}$.

Remark 3.6 The result is well-known for $\tau=0$, where (3.33) reads as $s_{1}-s_{2}>$ $d\left(\frac{1}{p_{1}}-\frac{1}{p_{2}}\right)_{+}$. We find it interesting that for $\tau>0$ there is not a simple ' $\tau$-shift', but an interplay between $\tau$ and the $p_{i}$-parameters - however, only when $p_{1} \geq p_{2}$. This again refers to the hybrid role played by the additional $\tau$-parameters and makes it even more obvious that it influences both the smoothness and the integrability parameters $s_{i}$ and $p_{i}$, respectively.

Now we deal with special target spaces. In the case of $L_{\infty}(\Omega)$ and $\operatorname{bmo}(\Omega)$ we have the following result.

Corollary 3.7 Let $s \in \mathbb{R}, 0<p \leq u<\infty$ and $q \in(0, \infty]$. Then the following conditions are equivalent: 
(i) the embedding $\mathcal{A}_{u, p, q}^{s}(\Omega) \hookrightarrow L_{\infty}(\Omega)$ is compact,

(ii) the embedding $\mathcal{A}_{u, p, q}^{s, p}(\Omega) \hookrightarrow \operatorname{bmo}(\Omega)$ is compact,

(iii) the inequality $s>\frac{d}{u}$ holds.

Proof The equivalence of (i) and (iii) was proved in [14,15], whereas the equivalence of (ii) and (iii) follows from Theorem 3.2 since $\operatorname{bmo}(\Omega)=F_{r, 2}^{0,1 / r}(\Omega), 0<r<\infty$, and $\mathcal{E}_{u, p, q}^{s}(\Omega)=F_{p, q}^{s, \tau}(\Omega), 0 \leq \tau=\frac{1}{p}-\frac{1}{u}$. This covers the case $\mathcal{A}_{u, p, q}^{s}=\mathcal{E}_{u, p, q}^{s}$. The extension to the case $\mathcal{A}_{u, p, q}^{s}=\mathcal{N}_{u, p, q}^{s}$ is done via (2.14).

The counterpart of Corollary 3.7 for spaces of type $A_{p, q}^{s, \tau}$ reads as follows.

Corollary 3.8 Let $s \in \mathbb{R}, \tau \geq 0,0<p, q \leq \infty$ (with $p<\infty$ in case of $A=F$ ). Then the following conditions are equivalent:

(i) the embedding $A_{p, q}^{s, \tau}(\Omega) \hookrightarrow L_{\infty}(\Omega)$ is compact,

(ii) the embedding $A_{p, q}^{s, \tau}(\Omega) \hookrightarrow \operatorname{bmo}(\Omega)$ is compact,

(iii) the inequality $s>d\left(\frac{1}{p}-\tau\right)$ holds.

Proof Step 1 We prove the equivalence of (i) and (iii). The case $\tau=0$ is well-known, so we assume $\tau>0$. Note that the continuity of that embedding was studied in [11, Proposition 2.18] already, with the outcome that $A_{p, q}^{s, \tau}(\Omega) \hookrightarrow L_{\infty}(\Omega)$ if, and only if, $s>d\left(\frac{1}{p}-\tau\right)$. Hence (i) implies (iii) and we are left to show the converse. Assume first, in addition, that $p \geq 2$, and $d\left(\frac{1}{p}-\tau\right)<s<d\left(\frac{1}{p}-\tau\right)+1$. We dealt with that situation in [43, Corollary 5.10] and characterized the asymptotic behaviour of approximation numbers of the embedding $A_{p, q}^{s, \tau}(\Omega) \hookrightarrow L_{\infty}(\Omega)$. In particular, that outcome implies (i). The additional restriction for $s$ (from above) can immediately be removed in view of (2.8) (adapted to spaces on bounded domains). Now let $0<p<2$ and $s>d\left(\frac{1}{p}-\tau\right)$. We use a Sobolev-type embedding: choose $\sigma=s-d\left(\frac{1}{p}-\frac{1}{2}\right)<s$, then $A_{2, q}^{\sigma, \tau}(\Omega) \hookrightarrow L_{\infty}(\Omega)$ compactly by our previous argument, and $A_{p, q}^{s, \tau}(\Omega) \hookrightarrow A_{2, q}^{\sigma, \tau}(\Omega)$ by the Sobolev-type embedding, cf. [43, Propositions 3.4, 3.8] (adapted to spaces on domains).

Step 2 We prove the equivalence of (ii) and (iii). However, in case of $A=F$ this coincides with Theorem 3.2 for $s_{1}=s, s_{2}=0, p_{1}=p_{2}=p, \tau_{1}=\tau, \tau_{2}=\frac{1}{p}$, $q_{1}=q$ and $q_{2}=2$ since $\operatorname{bmo}(\Omega)=F_{p, 2}^{0,1 / p}(\Omega)$. The extension to the case $A=B$ results from (2.8) and (2.10).

Remark 3.9 In case of $\tau>\frac{1}{p}$ or $\tau=\frac{1}{p}$ and $q=\infty$, Corollary 3.8 is well-known as a compact embedding within the scale of Besov spaces $B_{p, q}^{s}(\Omega)$, in view of Proposition 2.7. If $0 \leq \tau<\frac{1}{p}$, then Corollaries 3.7 and 3.8 coincide for $\mathcal{E}$-spaces, using (2.18). In view of our continuity result [11, Proposition 2.18] the above outcome can be reformulated for $\tau>0$ such that $A_{p, q}^{s, \tau}(\Omega) \hookrightarrow L_{\infty}(\Omega)$ is compact if, and only if, it is bounded. This is different from the case $\tau=0$.

We finally formulate the corresponding results for compact embeddings into $L_{r}(\Omega)$, $1 \leq r<\infty$, which can be seen as the counterparts of Corollaries 3.7 and 3.8 where $r=\infty$. 
Corollary 3.10 Let $s \in \mathbb{R}, 0<p, q \leq \infty$ (with $p<\infty$ in case of $A=F$ ), $1 \leq r<$ $\infty$.

(i) Let $u \in[p, \infty)($ or $p=u=\infty$ if $\mathcal{A}=\mathcal{N})$. Then

$$
\mathcal{A}_{u, p, q}^{s}(\Omega) \hookrightarrow L_{r}(\Omega) \text { is compact if, and only if, } s>\frac{d}{u}\left(1-\frac{p}{r}\right)_{+} .
$$

(ii) Let $\tau \geq 0$. Then

$$
A_{p, q}^{s, \tau}(\Omega) \hookrightarrow L_{r}(\Omega)
$$

is compact if, and only if,

$$
s>d \begin{cases}\left(\frac{1}{p}-\tau\right) & \text { if } \tau \geq \frac{1}{p}, \\ \left(\frac{1}{p}-\tau\right)\left(1-\frac{p}{r}\right)_{+} & \text {if } \tau \leq \frac{1}{p} .\end{cases}
$$

Proof Case (i) was already shown in [14, Proposition 5.3] (for $\mathcal{A}=\mathcal{N}$ ). In view of (2.14) and the independence of the condition with respect to $q$ the counterpart for $\mathcal{A}=\mathcal{E}$ follows (and slightly extends our recent result in [15, Corollary 5.4] to $r=1$ ). We come to (ii) and start with the case $A=B$. Note that $B_{r, 1}^{0}(\Omega) \hookrightarrow L_{r}(\Omega) \hookrightarrow$ $B_{r, \infty}^{0}(\Omega), 1 \leq r \leq \infty$, so we apply Theorem 3.2 for $s_{1}=s, s_{2}=0, p_{1}=p$, $p_{2}=r, \tau_{1}=\tau, \tau_{2}=0, q_{1}=q$, and $q_{2}=1$ or $q_{2}=\infty$ to obtain the necessary and sufficient conditions. Again we benefit from the independence of (3.10) with respect to the $q$-parameters. Finally, the case $A=F$ follows by (2.10) again.

Corollary 3.11 Let $s_{i} \in \mathbb{R}, 0<q_{i} \leq \infty, 0<p_{i} \leq \infty$ (with $p_{i}<\infty$ in case of $A=F), \tau_{i} \geq 0, i=1,2$. There is no continuous embedding

$$
\operatorname{id}_{\tau}: A_{p_{1}, q_{1}}^{s_{1}, \tau_{1}}(\Omega) \hookrightarrow A_{p_{2}, q_{2}}^{s_{2}, \tau_{2}}(\Omega)
$$

if

$$
\frac{s_{1}-s_{2}}{d}<\gamma\left(\tau_{1}, \tau_{2}, p_{1}, p_{2}\right)
$$

Proof Here we directly follow our proof of Theorem 3.2 and apply our continuity results [14, Theorem 3.1] (for $\mathcal{N}$-spaces) and [15, Theorem 5.2] (for $\mathcal{E}$-spaces). We again follow the splitting suggested by (3.11). So let us assume in the sequel that there is a continuous embedding $\mathrm{id}_{\tau}$.

Step 1 Let $\tau_{2} \geq \frac{1}{p_{2}}$ with $q_{2}=\infty$ if $\tau_{2}=\frac{1}{p_{2}}$. If also $\tau_{1} \geq \frac{1}{p_{1}}$ with $q_{1}=\infty$ if $\tau_{1}=\frac{1}{p_{2}}$, then (3.14) implies the continuity of

$$
\operatorname{id}: B_{\infty, \infty}^{s_{1}+d\left(\tau_{1}-\frac{1}{p_{1}}\right)}(\Omega) \hookrightarrow B_{\infty, \infty}^{s_{2}+d\left(\tau_{2}-\frac{1}{p_{2}}\right)}(\Omega)
$$


which is well-known to imply

$$
s_{1}+d\left(\tau_{1}-\frac{1}{p_{1}}\right) \geq s_{2}+d\left(\tau_{2}-\frac{1}{p_{2}}\right),
$$

contradicting (3.35) in that case. Likewise, if $\tau_{1} \leq \frac{1}{p_{1}}$ with $q_{1}<\infty$ if $\tau_{1}=\frac{1}{p_{1}}$, then (3.15) leads to the continuity of

$$
\mathrm{id}: B_{p_{0}, q_{0}}^{s_{1}+d\left(\tau_{1}-\frac{1}{p_{1}}+\frac{1}{p_{0}}\right)}(\Omega) \hookrightarrow B_{\infty, \infty}^{s_{2}+d\left(\tau_{2}-\frac{1}{p_{2}}\right)}
$$

which implies again (3.36) and thus contradicts (3.35).

Step 2 Assume $\tau_{2}<\frac{1}{p_{2}}$ and proceed parallel to Step 2 of the proof of Theorem 3.2. If also $\tau_{1}<\frac{1}{p_{1}}$, then the continuity of $\operatorname{id}_{\tau}: F_{p_{1}, q_{1}}^{s_{1}, \tau_{1}}(\Omega) \hookrightarrow F_{p_{2}, q_{2}}^{s_{2}, \tau_{2}}(\Omega)$ results in the continuity of the corresponding embedding between $\mathcal{E}$-spaces, using (2.18), which in turn by [15, Theorem 5.2] leads to a contradiction of (3.35) again. The extension to spaces $B_{p, q}^{s, \tau}(\Omega)$ is obtained via (2.16), (2.17),

$$
\mathcal{N}_{u_{1}, p_{1}, q_{1}}^{s_{1}}(\Omega) \hookrightarrow B_{p_{1}, q_{1}}^{s_{1}, \tau_{1}}(\Omega) \hookrightarrow B_{p_{2}, q_{2}}^{s_{2}, \tau_{2}}(\Omega) \hookrightarrow B_{p_{2}, \infty}^{s_{2}, \tau_{2}}(\Omega)=\mathcal{N}_{u_{2}, p_{2}, \infty}^{s_{2}}(\Omega),
$$

which by [14, Theorem 3.1] implies

$$
\frac{s_{1}-s_{2}}{d} \geq \max \left\{0, \frac{1}{p_{1}}-\tau_{1}-\frac{1}{p_{2}}+\max \left\{\tau_{2}, \frac{p_{1}}{p_{2}} \tau_{1}\right\}\right\}=\gamma\left(\tau_{1}, \tau_{2}, p_{1}, p_{2}\right)
$$

contradicting (3.35) in this setting. If $\tau_{1} \geq \frac{1}{p_{1}}$ with $q_{1}=\infty$ when $\tau_{1}=\frac{1}{p_{1}}$, then Proposition 2.7 together with (2.17), (2.18), (2.14) yield the continuity of the outer embedding in the following chain:

$\mathcal{N}_{\infty, \infty, \infty}^{s_{1}+d\left(\tau_{1}-\frac{1}{p_{1}}\right)}(\Omega)=B_{\infty, \infty}^{s_{1}+d\left(\tau_{1}-\frac{1}{p_{1}}\right)}(\Omega)=A_{p_{1}, q_{1}}^{s_{1}, \tau_{1}}(\Omega) \hookrightarrow A_{p_{2}, q_{2}}^{s_{2}, \tau_{2}}(\Omega) \hookrightarrow \mathcal{N}_{u_{2}, p_{2}, \infty}^{s_{2}}(\Omega)$

Thus we have again $s_{1}-s_{2} \geq d\left(\frac{1}{p_{1}}-\tau_{1}\right)$, contradicting (3.35). When $\tau_{1}=\frac{1}{p_{1}}$, $q_{1}<\infty$, (3.23) leads to the same contradiction again.

Step 3 Assume finally $\tau_{2}=\frac{1}{p_{2}}, q_{2}<\infty$. Then the embeddings (3.25), (3.27) and (3.29) disprove (3.35) in the corresponding settings.

Remark 3.12 Obviously Theorem 3.2 implies, in particular, that the embedding $\mathrm{id}_{\tau}$ is continuous when $s_{1}-s_{2}>d \gamma\left(\tau_{1}, \tau_{2}, p_{1}, p_{2}\right)$. In view of Corollary 3.11 it thus turns out that the limiting case for the embedding $\mathrm{id}_{\tau}$ is indeed

$$
\frac{s_{1}-s_{2}}{d}=\gamma\left(\tau_{1}, \tau_{2}, p_{1}, p_{2}\right)
$$

Here some influence of the fine parameters $q_{i}$ can also be expected. But this question is postponed to a separate study in the future. 


\section{Entropy numbers}

First we return to the compact embedding $\operatorname{id}_{\mathcal{A}}$ given by (3.1), recall Theorem 3.1. For its entropy numbers we obtained in [17, Corollaries $4.1,4.3]$ the following result.

Theorem 4.1 Let $s_{i} \in \mathbb{R}, 0<q_{i} \leq \infty, 0<p_{i} \leq u_{i}<\infty$ or, in the case of $\mathcal{N}$-spaces, allow also $p_{i}=u_{i}=\infty, i=1,2$. Assume that (3.2) is satisfied. Then we obtain for the entropy numbers of the compact embedding

$$
\operatorname{id}_{\mathcal{A}}: \mathcal{A}_{u_{1}, p_{1}, q_{1}}^{s_{1}}(\Omega) \hookrightarrow \mathcal{A}_{u_{2}, p_{2}, q_{2}}^{s_{2}}(\Omega)
$$

the following results:

(i) If

$$
\frac{1}{p_{1}}-\frac{1}{p_{2}} \geq \frac{s_{1}-s_{2}}{d}>\frac{p_{1}}{u_{1}}\left(\frac{1}{p_{1}}-\frac{1}{p_{2}}\right) \quad \text { and } \quad \frac{u_{2}}{p_{2}}<\frac{u_{1}}{p_{1}},
$$

then there exists some $c>0$ and for any $\varepsilon>0$ some $c_{\varepsilon}>0$ such that for all $k \in \mathbb{N}$,

$$
c k^{-\frac{u_{1}}{u_{1}-p_{1}}\left(\frac{s_{1}-s_{2}}{d}-\frac{p_{1}}{u_{1}}\left(\frac{1}{p_{1}}-\frac{1}{p_{2}}\right)\right)} \leq e_{k}\left(\operatorname{id}_{\mathcal{A}}\right) \leq c_{\varepsilon} k^{-\frac{u_{1}}{u_{1}-p_{1}}\left(\frac{s_{1}-s_{2}}{d}-\frac{p_{1}}{u_{1}}\left(\frac{1}{p_{1}}-\frac{1}{p_{2}}\right)\right)+\varepsilon} .
$$

(ii) In all other cases admitted by (3.2), it holds

$$
e_{k}\left(\operatorname{id}_{\mathcal{A}}: \mathcal{A}_{u_{1}, p_{1}, q_{1}}^{s_{1}}(\Omega) \hookrightarrow \mathcal{A}_{u_{2}, p_{2}, q_{2}}^{s_{2}}(\Omega)\right) \sim k^{-\frac{s_{1}-s_{2}}{d}}, \quad k \in \mathbb{N} .
$$

Proof The cases when $0<p_{i} \leq u_{i}<\infty, i=1,2$, were proved in [17], as already mentioned. So it remains to verify the cases $p_{1}=u_{1}=\infty$ or $p_{2}=u_{2}=\infty$. Note that in both cases we are in part (ii).

If $p_{1}=u_{1}=\infty,(3.2)$ reads as $s_{1}-s_{2}>0$. Now we use (3.3) and the multiplicativity of the entropy numbers to obtain

$$
e_{k}\left(\operatorname{id}_{\mathcal{A}}\right) \lesssim e_{k}\left(B_{\infty, q_{1}}^{s_{1}}(\Omega) \hookrightarrow A_{u_{2}, q_{2}}^{s_{2}}(\Omega)\right) \lesssim k^{-\frac{s_{1}-s_{2}}{d}}
$$

Moreover, by (3.4) we get the desired estimate from below:

$$
e_{k}\left(\operatorname{id}_{\mathcal{A}}\right) \gtrsim e_{k}\left(B_{\infty, q_{1}}^{s_{1}}(\Omega) \hookrightarrow A_{p_{2}, q_{2}}^{s_{2}}(\Omega)\right) \gtrsim k^{-\frac{s_{1}-s_{2}}{d}}
$$

The case when $p_{2}=u_{2}=\infty$ and $\frac{s_{1}-s_{2}}{d}>\frac{1}{u_{1}}$ follows similarly but using (3.5)-(3.7) this time.

Now we give the counterpart of the above result for the compact embedding (3.9), described by Theorem 3.2. 
Theorem 4.2 Let $s_{i} \in \mathbb{R}, 0<q_{i} \leq \infty, 0<p_{i} \leq \infty$ (with $p_{i}<\infty$ in case of $A=F), \tau_{i} \geq 0, i=1,2$. Assume that (3.10) is satisfied. Then we obtain for the entropy numbers of the compact embedding

$$
\operatorname{id}_{\tau}: A_{p_{1}, q_{1}}^{s_{1}, \tau_{1}}(\Omega) \hookrightarrow A_{p_{2}, q_{2}}^{s_{2}, \tau_{2}}(\Omega)
$$

the following results:

(i) If $\tau_{1}<\frac{1}{p_{1}}$,

$$
\tau_{1} \frac{p_{1}}{p_{2}}>\tau_{2}
$$

and

$$
\frac{1}{p_{1}}-\frac{1}{p_{2}} \geq \frac{s_{1}-s_{2}}{d}>\left(1-p_{1} \tau_{1}\right)\left(\frac{1}{p_{1}}-\frac{1}{p_{2}}\right),
$$

then there exists some $c>0$ and for any $\varepsilon>0$ some $c_{\varepsilon}>0$ such that for all $k \in \mathbb{N}$,

$$
c k^{-\frac{1}{p_{1} \tau_{1}}\left(\frac{s_{1}-s_{2}}{d}-\left(1-p_{1} \tau_{1}\right)\left(\frac{1}{p_{1}}-\frac{1}{p_{2}}\right)\right)} \leq e_{k}\left(\mathrm{id}_{\tau}\right) \leq c_{\varepsilon} k^{-\frac{1}{p_{1} \tau_{1}}\left(\frac{s_{1}-s_{2}}{d}-\left(1-p_{1} \tau_{1}\right)\left(\frac{1}{p_{1}}-\frac{1}{p_{2}}\right)\right)+\varepsilon} .
$$

(ii) In all other cases admitted by (3.10), it holds

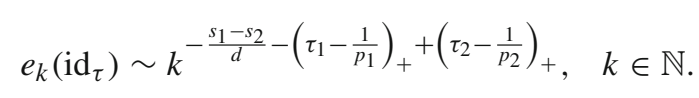

Proof To prove this result we basically rely on the proof of Theorem 3.2 and the corresponding counterparts of the entropy numbers for the spaces $\mathcal{A}_{u, p, q}^{s}$, cf. Theorem 4.1, as well as for the classical spaces $A_{p, q}^{s}$, cf. Remark 2.15. Therefore, we start by proving part (i) and then we split part (ii) in all the possible cases as in the proof of Theorem 3.2.

Step 1 We deal with case (i). Assumption (4.4) implies also $\tau_{2}<\frac{1}{p_{2}}$, such that in view of our reformulation (3.11) of the compactness condition (3.10) it is obvious that the expression on the right-hand side of (3.10) equals $\left(1-p_{1} \tau_{1}\right)\left(\frac{1}{p_{1}}-\frac{1}{p_{2}}\right)_{+}$. We first use (2.18) to get

$$
\begin{gathered}
e_{k}\left(\operatorname{id}_{\tau}: F_{p_{1}, q_{1}}^{s_{1}, \tau_{1}}(\Omega) \hookrightarrow F_{p_{2}, q_{2}}^{s_{2}, \tau_{2}}(\Omega)\right) \sim e_{k}\left(\operatorname{id}_{\mathcal{E}}: \mathcal{E}_{u_{1}, p_{1}, q_{1}}^{s_{1}}(\Omega) \hookrightarrow \mathcal{E}_{u_{2}, p_{2}, q_{2}}^{s_{2}}(\Omega)\right), \\
\frac{1}{u_{i}}=\frac{1}{p_{i}}-\tau_{i}, \quad i=1,2,
\end{gathered}
$$

together with Theorem 4.1(i) which covers the case $A=F$. As for the $B$-case, we benefit from (3.17) (to the estimate from above) and (3.18) (to the estimate from below), such that the multiplicativity of entropy numbers implies

$$
\begin{aligned}
e_{k}\left(\operatorname{id}_{\mathcal{N}}: \mathcal{N}_{u_{1}, p_{1}, q_{1}}^{s_{1}}(\Omega) \hookrightarrow \mathcal{N}_{u_{2}, p_{2}, \infty}^{s_{2}}(\Omega)\right) & \lesssim e_{k}\left(\operatorname{id}_{\tau}\right) \\
& \lesssim e_{k}\left(\operatorname{id}_{\mathcal{N}}: \mathcal{N}_{u_{1}, p_{1}, \infty}^{s_{1}}(\Omega) \hookrightarrow \mathcal{N}_{u_{2}, p_{2}, q_{2}}^{s_{2}}(\Omega)\right)
\end{aligned}
$$


Application of Theorem 4.1(i) concludes the argument.

Step 2 We are left to prove (4.7) in all remaining cases.

Substep 2.1 We first continue with the case $\tau_{i}<\frac{1}{p_{i}}, i=1,2$, from Step 1, where we now assume that (4.4) or (4.5) are not satisfied. In both cases we proceed as above and use the coincidence (2.18) together with Theorem 4.1(ii). This yields

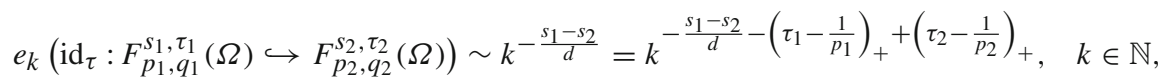

i.e. the desired result (4.7) for $A=F$. The case $A=B$ is done as in the end of Step 1, using again (3.17) and (3.18).

Substep 2.2 We stick to $\tau_{2}<\frac{1}{p_{2}}$, now together with $\tau_{1} \geq \frac{1}{p_{1}}$ and $q_{1}=\infty$ if $\tau_{1}=\frac{1}{p_{1}}$. In view of (3.11) we thus assume $s_{1}-s_{2}>d\left(\frac{1}{p_{1}}-\tau_{1}\right)$ and need to show (4.7) in the form

$$
e_{k}\left(\mathrm{id}_{\tau}\right) \sim k^{-\frac{s_{1}-s_{2}}{d}-\left(\tau_{1}-\frac{1}{p_{1}}\right)}, \quad k \in \mathbb{N}
$$

This can be seen as follows. By (3.21),

$$
e_{k}\left(\operatorname{id}_{\tau}: A_{p_{1}, q_{1}}^{s_{1}, \tau_{1}}(\Omega) \hookrightarrow A_{p_{2}, q_{2}}^{s_{2}, \tau_{2}}(\Omega)\right) \gtrsim e_{k}\left(\operatorname{id}_{\mathcal{N}}: \mathcal{N}_{\infty, \infty, \infty}^{s_{1}+d\left(\tau_{1}-\frac{1}{p_{1}}\right)}(\Omega) \hookrightarrow \mathcal{N}_{u_{2}, p_{2}, \infty}^{s_{2}}(\Omega)\right),
$$

which in view of Theorem 4.1 (applied to $\mathcal{A}=\mathcal{N}$ ) leads to

$$
e_{k}\left(\operatorname{id}_{\tau}: A_{p_{1}, q_{1}}^{s_{1}, \tau_{1}}(\Omega) \hookrightarrow A_{p_{2}, q_{2}}^{s_{2}, \tau_{2}}(\Omega)\right) \gtrsim k^{-\frac{s_{1}-s_{2}}{d}-\left(\tau_{1}-\frac{1}{p_{1}}\right)}, \quad k \in \mathbb{N},
$$

under the given assumptions. The estimate from above follows similarly from (3.20) and again the multiplicativity of entropy numbers.

Substep 2.3 We study the case $\tau_{2} \geq \frac{1}{p_{2}}$ with $q_{2}=\infty$ if $\tau_{2}=\frac{1}{p_{2}}$. According to (3.11) we assume that

$$
\frac{s_{1}-s_{2}}{d}>\frac{1}{p_{1}}-\tau_{1}-\frac{1}{p_{2}}+\tau_{2} .
$$

If $\tau_{1} \geq \frac{1}{p_{1}}$ with $q_{1}=\infty$ if $\tau_{1}=\frac{1}{p_{1}}$, then Proposition 2.7 implies that $e_{k}\left(\operatorname{id}_{\tau}\right) \sim e_{k}\left(\operatorname{id}_{B}: B_{\infty, \infty}^{\sigma_{1}}(\Omega) \hookrightarrow B_{\infty, \infty}^{\sigma_{2}}(\Omega)\right), \quad \sigma_{i}=s_{i}+d\left(\tau_{i}-\frac{1}{p_{i}}\right), \quad i=1,2$.

But the asymptotic behaviour of the entropy numbers in that latter case is well-known for $\sigma_{1}>\sigma_{2}$, which is equivalent to (4.8), that is

$$
e_{k}\left(\operatorname{id}_{B}: B_{\infty, \infty}^{\sigma_{1}}(\Omega) \hookrightarrow B_{\infty, \infty}^{\sigma_{2}}(\Omega)\right) \sim k^{-\frac{\sigma_{1}-\sigma_{2}}{d}}, \quad k \in \mathbb{N}
$$


cf. Remark 2.15. This coincides with (4.7) in that case.

If $\tau_{1}<\frac{1}{p_{1}}$ we argue as follows. We use (2.18), (2.6) and Proposition 2.7 to get

$$
e_{k}\left(\operatorname{id}_{\tau}: F_{p_{1}, q_{1}}^{s_{1}, \tau_{1}}(\Omega) \hookrightarrow F_{p_{2}, q_{2}}^{s_{2}, \tau_{2}}(\Omega)\right) \sim e_{k}\left(\mathrm{id}: \mathcal{E}_{u_{1}, p_{1}, q_{1}}^{s_{1}}(\Omega) \hookrightarrow \mathcal{N}_{\infty, \infty, \infty}^{s_{2}+d\left(\tau_{2}-\frac{1}{p_{2}}\right)}(\Omega)\right)
$$

On the other hand, Theorem 4.1(ii) yields

$$
e_{k}\left(\operatorname{id}_{\mathcal{N}}: \mathcal{N}_{u_{1}, p_{1}, q_{1}}^{s_{1}}(\Omega) \hookrightarrow \mathcal{N}_{\infty, \infty, \infty}^{s_{2}+d\left(\tau_{2}-\frac{1}{p_{2}}\right)}(\Omega)\right) \sim k^{-\frac{s_{1}-s_{2}}{d}+\tau_{2}-\frac{1}{p_{2}}}, \quad k \in \mathbb{N},
$$

whenever (4.8) is satisfied. In view of (2.14) we can thus continue (4.9) by

$$
e_{k}\left(\operatorname{id}_{\tau}: F_{p_{1}, q_{1}}^{s_{1}, \tau_{1}}(\Omega) \hookrightarrow F_{p_{2}, q_{2}}^{s_{2}, \tau_{2}}(\Omega)\right) \sim k^{-\frac{s_{1}-s_{2}}{d}+\tau_{2}-\frac{1}{p_{2}}}, \quad k \in \mathbb{N},
$$

which is the desired result in case of $A=F$. The case $A=B$ can be obtained noting that

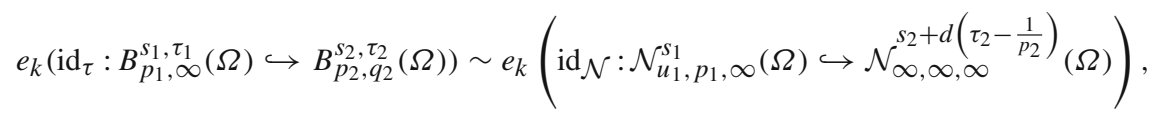

where we have used (2.6) and (2.17). Now (2.9) and Theorem 4.1(ii) give the desired result.

Step 3 It remains to study the limiting cases, that is, when $\tau_{i}=\frac{1}{p_{i}}$ and $q_{i}<\infty$ for $i=1$ or $i=2$.

Substep 3.1 Let $\tau_{1}=\frac{1}{p_{1}}, q_{1}<\infty$, and $\tau_{2}<\frac{1}{p_{2}}$. Following the arguments of Substep 2.3 of the proof of Theorem 3.2, in particular, (3.22) and (3.23), and using the multiplicativity of entropy numbers we arrive at

$$
e_{k}\left(\mathrm{id}: B_{\infty, q_{1}}^{s_{1}}(\Omega) \hookrightarrow \mathcal{N}_{u_{2}, p_{2}, \infty}^{s_{2}}(\Omega)\right) \lesssim e_{k}\left(\mathrm{id}_{\tau}\right) \lesssim e_{k}\left(\mathrm{id}: B_{\infty, \infty}^{s_{1}}(\Omega) \hookrightarrow \mathcal{A}_{u_{2}, p_{2}, q_{2}}^{s_{2}}(\Omega)\right)
$$

where $\frac{1}{u_{2}}=\frac{1}{p_{2}}-\tau_{2}>0$. However, completely parallel to Substeps 2.2 and 2.3, Theorem 4.1(ii) implies

$$
e_{k}\left(\mathrm{id}: B_{\infty, \infty}^{s_{1}}(\Omega) \hookrightarrow \mathcal{A}_{u_{2}, p_{2}, q_{2}}^{s_{2}}(\Omega)\right) \sim e_{k}\left(\mathrm{id}: B_{\infty, q_{1}}^{s_{1}}(\Omega) \hookrightarrow \mathcal{N}_{u_{2}, p_{2}, \infty}^{s_{2}}(\Omega)\right) \sim k^{-\frac{s_{1}-s_{2}}{d}}
$$

whenever $s_{1}>s_{2}$, such that (4.10) finally results in

$$
e_{k}\left(\operatorname{id}_{\tau}\right) \sim k^{-\frac{s_{1}-s_{2}}{d}}, \quad k \in \mathbb{N} .
$$


Substep 3.2 Let $\tau_{1}=\frac{1}{p_{1}}, q_{1}<\infty$, and $\tau_{2} \geq \frac{1}{p_{2}}$ with $q_{2}=\infty$ if $\tau_{2}=\frac{1}{p_{2}}$. In view of (3.11), we assume now $s_{1}-s_{2}>d\left(\tau_{2}-\frac{1}{p_{2}}\right)$. Then (3.13) leads to

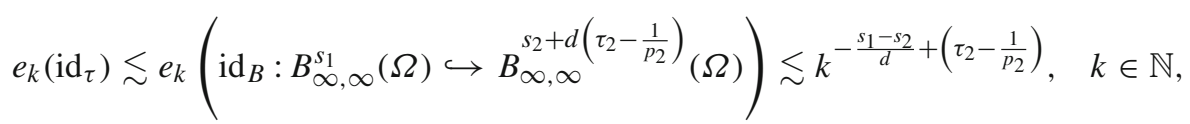

by the same arguments as above. Conversely, according to [44, Corollary 5.2] (adapted to spaces on bounded domains), Proposition 2.7 and (2.10), we have in this case

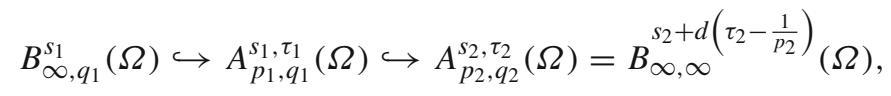

such that

$e_{k}\left(\operatorname{id}_{\tau}\right) \gtrsim e_{k}\left(\operatorname{id}_{B}: B_{\infty, q_{1}}^{s_{1}}(\Omega) \hookrightarrow B_{\infty, \infty}^{s_{2}+d\left(\tau_{2}-\frac{1}{p_{2}}\right)}(\Omega)\right) \gtrsim k^{-\frac{s_{1}-s_{2}}{d}+\left(\tau_{2}-\frac{1}{p_{2}}\right)}, \quad k \in \mathbb{N}$

This concludes the proof in this case.

Substep 3.3 Let $\tau_{2}=\frac{1}{p_{2}}, q_{2}<\infty$, and assume $\tau_{1}<\frac{1}{p_{1}}$. The chain of embeddings (3.25) leads to

$$
e_{k}\left(\mathrm{id}_{\tau}\right) \gtrsim e_{k}\left(\mathrm{id}: A_{u_{1}, q_{1}}^{s_{1}}(\Omega) \hookrightarrow B_{\infty, \infty}^{s_{2}}(\Omega)\right) \gtrsim k^{-\frac{s_{1}-s_{2}}{d}}, \quad k \in \mathbb{N},
$$

as desired, where we made use of the condition $\frac{s_{1}-s_{2}}{d}>\frac{1}{u_{1}}=\frac{1}{p_{1}}-\tau_{1}$ as (3.11) reads in this case.

For the estimate from above we use (3.24). In the same way as there, if $A=B$ we take $q_{0}=q_{2}$, and in case $A=F$ we choose $q_{0} \leq \min \left\{p_{2}, q_{2}\right\}$ and further use (2.10). Thus

$e_{k}\left(\operatorname{id}_{\tau}: A_{p_{1}, q_{1}}^{s_{1}, \tau_{1}}(\Omega) \hookrightarrow A_{p_{2}, q_{2}}^{s_{2}, \tau_{2}}(\Omega)\right) \lesssim e_{k}\left(\operatorname{id}: \mathcal{N}_{u_{1}, p_{1}, \infty}^{s_{1}}(\Omega) \hookrightarrow \mathcal{N}_{\infty, \infty, q_{0}}^{s_{2}}(\Omega)\right) \lesssim k^{-\frac{s_{1}-s_{2}}{d}}, \quad k \in \mathbb{N}$,

by Theorem 4.1(ii).

Substep 3.4 Let $\tau_{2}=\frac{1}{p_{2}}, q_{2}<\infty$, and $\tau_{1} \geq \frac{1}{p_{1}}$ with $q_{1}=\infty$ if $\tau_{1}=\frac{1}{p_{1}}$. Assume that $s_{1}-s_{2}>d\left(\tau_{1}-\frac{1}{p_{1}}\right)$. We benefit from (3.27) to conclude that

$e_{k}\left(\operatorname{id}_{\tau}\right) \gtrsim e_{k}\left(\operatorname{id}_{B}: B_{\infty, \infty}^{s_{1}+d\left(\tau_{1}-\frac{1}{p_{1}}\right)}(\Omega) \hookrightarrow B_{\infty, \infty}^{s_{2}}(\Omega)\right) \gtrsim k^{-\frac{s_{1}-s_{2}}{d}-\left(\tau_{1}-\frac{1}{p_{1}}\right)}, \quad k \in \mathbb{N}$.

For the estimate from above we adapt (3.24) properly by

$$
A_{p_{1}, q_{1}}^{s_{1}, \tau_{1}}(\Omega)=B_{\infty, \infty}^{s_{1}+d\left(\tau_{1}-\frac{1}{p_{1}}\right)}(\Omega)=\mathcal{N}_{\infty, \infty, \infty}^{s_{1}+d\left(\tau_{1}-\frac{1}{p_{1}}\right)}(\Omega) \hookrightarrow \mathcal{N}_{\infty, \infty, q_{0}}^{s_{2}}(\Omega)=B_{\infty, q_{0}}^{s_{2}}(\Omega) \hookrightarrow A_{p_{2}, q_{2}}^{s_{2}, \tau_{2}}(\Omega),
$$


with the same choice of $q_{0}$ as before. Then we get

$e_{k}\left(\operatorname{id}_{\tau}\right) \lesssim e_{k}\left(\operatorname{id}_{\mathcal{N}}: \mathcal{N}_{\infty, \infty, \infty}^{s_{1}+d\left(\tau_{1}-\frac{1}{p_{1}}\right)}(\Omega) \hookrightarrow \mathcal{N}_{\infty, \infty, q_{2}}^{s_{2}}(\Omega)\right) \lesssim k^{-\frac{s_{1}-s_{2}}{d}-\left(\tau_{1}-\frac{1}{p_{1}}\right)}, \quad k \in \mathbb{N}$

Substep 3.5 In the double-limiting case, that is, when $\tau_{i}=\frac{1}{p_{i}}, q_{i}<\infty, i=1,2$, then (3.28) and (3.29) immediately imply

$$
e_{k}\left(\mathrm{id}_{\tau}\right) \sim k^{-\frac{s_{1}-s_{2}}{d}}, \quad k \in \mathbb{N}
$$

since

$e_{k}\left(\operatorname{id}_{B}: B_{\infty, \infty}^{s_{1}}(\Omega) \hookrightarrow B_{\infty, q_{2}}^{s_{2}}(\Omega)\right) \sim e_{k}\left(\operatorname{id}_{B}: B_{\infty, q_{0}}^{s_{1}}(\Omega) \hookrightarrow B_{\infty, \infty}^{s_{2}}(\Omega)\right) \sim k^{-\frac{s_{1}-s_{2}}{d}}$

whenever $s_{1}>s_{2}$, cf. Remark 2.15.

Remark 4.3 We return to our Remarks 2.9 and 3.4 and formulate the result for Triebel's hybrid spaces $L^{r} A_{p, q}^{s}(\Omega)$. Let $s_{i} \in \mathbb{R}, 0<q_{i} \leq \infty, 0<p_{i}<\infty,-\frac{d}{p_{i}} \leq r_{i}<\infty$, $i=1,2$, satisfy (3.31). Then we obtain for the compact embedding

$$
\operatorname{id}_{L}: L^{r_{1}} A_{p_{1}, q_{1}}^{s_{1}}(\Omega) \hookrightarrow L^{r_{2}} A_{p_{2}, q_{2}}^{s_{2}}(\Omega)
$$

the following results:

(i) If $r_{1}<0, r_{1} p_{1}>r_{2} p_{2}$, and

$$
\frac{1}{p_{1}}-\frac{1}{p_{2}} \geq \frac{s_{1}-s_{2}}{d}>-\frac{p_{1} r_{1}}{d}\left(\frac{1}{p_{1}}-\frac{1}{p_{2}}\right),
$$

then there exists some $c>0$ and for any $\varepsilon>0$ some $c_{\varepsilon}>0$ such that for all $k \in \mathbb{N}$,

$$
c k^{-\frac{1}{d+p_{1} r_{1}}\left(s_{1}-s_{2}+p_{1} r_{1}\left(\frac{1}{p_{1}}-\frac{1}{p_{2}}\right)\right)} \leq e_{k}\left(\mathrm{id}_{L}\right) \leq c_{\varepsilon} k^{-\frac{1}{d+p_{1} r_{1}}\left(s_{1}-s_{2}+p_{1} r_{1}\left(\frac{1}{p_{1}}-\frac{1}{p_{2}}\right)\right)+\varepsilon} .
$$

(ii) In all other cases admitted by (3.31), it holds

$$
e_{k}\left(\operatorname{id}_{L}\right) \sim k^{-\frac{s_{1}-s_{2}}{d}-\frac{\left(r_{1}\right)_{+}}{d}+\frac{\left(r_{2}\right)_{+}}{d}}, \quad k \in \mathbb{N}
$$

Parallel to the end of Sect. 3 we now collect some consequences and special cases of Theorem 4.2. We begin with the counterpart of Corollary 3.5, that is, when $\tau_{1}=$ $\tau_{2} \geq 0$.

Corollary 4.4 Let $s_{i} \in \mathbb{R}, 0<q_{i} \leq \infty, 0<p_{i} \leq \infty$ (with $p_{i}<\infty$ in case of $A=F), i=1,2$, and $\tau \geq 0$. Assume that (3.33) is satisfied. Then we obtain for the entropy numbers of the compact embedding

$$
\operatorname{id}_{\tau}: A_{p_{1}, q_{1}}^{s_{1}, \tau}(\Omega) \hookrightarrow A_{p_{2}, q_{2}}^{s_{2}, \tau}(\Omega)
$$


that

$$
e_{k}\left(\operatorname{id}_{\tau}: A_{p_{1}, q_{1}}^{s_{1}, \tau}(\Omega) \hookrightarrow A_{p_{2}, q_{2}}^{s_{2}, \tau}(\Omega)\right) \sim k^{-\frac{s_{1}-s_{2}}{d}-\left(\tau-\frac{1}{p_{1}}\right)_{+}+\left(\tau-\frac{1}{p_{2}}\right)_{+}}, \quad k \in \mathbb{N} .
$$

Proof This follows immediately from an application of Theorem 4.2 with $\tau_{1}=\tau_{2}=\tau$. Note that part (i) of Theorem 4.2 cannot appear in this setting.

Remark 4.5 The above result is again well-known for $\tau=0$. We find it interesting to note that for sufficiently small $\tau$, that is, when $0 \leq \tau \leq \min \left\{\frac{1}{p_{1}}, \frac{1}{p_{2}}\right\}$, the asymptotic behaviour for the entropy numbers remains the same,

$$
e_{k}\left(\operatorname{id}_{A}: A_{p_{1}, q_{1}}^{s_{1}}(\Omega) \hookrightarrow A_{p_{2}, q_{2}}^{s_{2}}(\Omega)\right) \sim e_{k}\left(\operatorname{id}_{\tau}: A_{p_{1}, q_{1}}^{s_{1}, \tau}(\Omega) \hookrightarrow A_{p_{2}, q_{2}}^{s_{2}, \tau}(\Omega)\right) \sim k^{-\frac{s_{1}-s_{2}}{d}} .
$$

For sufficiently large $\tau$, also the $\tau$-dependence disappears, due to the coincidence stated in Proposition 2.7, that is,

$$
e_{k}\left(\operatorname{id}_{\tau}: A_{p_{1}, q_{1}}^{s_{1}, \tau}(\Omega) \hookrightarrow A_{p_{2}, q_{2}}^{s_{2}, \tau}(\Omega)\right) \sim k^{-\frac{s_{1}-s_{2}}{d}+\frac{1}{p_{1}}-\frac{1}{p_{2}}}, \quad \tau \geq \max \left\{\frac{1}{p_{1}}, \frac{1}{p_{2}}\right\} .
$$

So only 'in between', that is, for $\min \left\{\frac{1}{p_{1}}, \frac{1}{p_{2}}\right\}<\tau<\max \left\{\frac{1}{p_{1}}, \frac{1}{p_{2}}\right\}$ the Morrey parameter $\tau$ influences the asymptotic behaviour of entropy numbers. Plainly, for $p_{1}=p_{2}$ this case is impossible, so in that case we would really have some ' $\tau$-shift',

$$
e_{k}\left(\operatorname{id}_{A}: A_{p, q_{1}}^{s_{1}}(\Omega) \hookrightarrow A_{p, q_{2}}^{s_{2}}(\Omega)\right) \sim e_{k}\left(\operatorname{id}_{\tau}: A_{p, q_{1}}^{s_{1}, \tau}(\Omega) \hookrightarrow A_{p, q_{2}}^{s_{2}, \tau}(\Omega)\right) \sim k^{-\frac{s_{1}-s_{2}}{d}}, \quad \tau \geq 0 .
$$

We come to the counterpart of Corollary 3.7.

Corollary 4.6 Let $s \in \mathbb{R}, 0<p \leq u<\infty, q \in(0, \infty]$, and $s>\frac{d}{u}$. Then the entropy numbers of the compact embeddings $\mathrm{id}: \mathcal{A}_{u, p, q}^{s}(\Omega) \hookrightarrow L_{\infty}(\Omega)$ and id: $\mathcal{A}_{u, p, q}^{s}(\Omega) \hookrightarrow \operatorname{bmo}(\Omega)$ behave like

$e_{k}\left(\operatorname{id}: \mathcal{A}_{u, p, q}^{s}(\Omega) \hookrightarrow L_{\infty}(\Omega)\right) \sim e_{k}\left(\mathrm{id}: \mathcal{A}_{u, p, q}^{s}(\Omega) \hookrightarrow \operatorname{bmo}(\Omega)\right) \sim k^{-\frac{s}{d}}, \quad k \in \mathbb{N}$.

Proof We can apply part (ii) of Theorem 4.2 due to the coincidence $\operatorname{bmo}(\Omega)=$ $F_{r, 2}^{0,1 / r}(\Omega), 0<r<\infty$, and the well-known embeddings $B_{\infty, 1}^{0}(\Omega) \hookrightarrow L_{\infty}(\Omega) \hookrightarrow$ $B_{\infty, \infty}^{0}(\Omega)$, i.e. we work with the assumption $\tau_{1} p_{1}<1=\tau_{2} p_{2}$ or $\tau_{2}=\frac{1}{p_{2}}=0$.

Step 1 Let us first consider the embedding $\mathcal{A}_{u, p, q}^{s}(\Omega) \hookrightarrow \operatorname{bmo}(\Omega)$. We get the result for $\mathcal{A}=\mathcal{E}$ by applying Theorem 4.2(ii) with $\mathcal{E}_{u, p, q}^{s, q}(\Omega)=F_{p, q}^{s, \tau}(\Omega), 0 \leq \tau=\frac{1}{p}-\frac{1}{u}$, that is $e_{k}\left(\operatorname{id}: \mathcal{E}_{u, p, q}^{s}(\Omega) \hookrightarrow \operatorname{bmo}(\Omega)\right) \sim e_{k}\left(\operatorname{id}_{\tau}: F_{p, q}^{s, \tau}(\Omega) \hookrightarrow F_{r, 2}^{0, \frac{1}{r}}(\Omega)\right) \sim k^{-\frac{s}{d}}, \quad k \in \mathbb{N}$. 
As for the case $\mathcal{A}=\mathcal{N}$, we use (2.17), (2.19) and (2.6) and obtain for $k \in \mathbb{N}$

$$
\begin{aligned}
& e_{k}\left(\operatorname{id}_{\tau}: B_{u, q}^{s, 0}(\Omega) \hookrightarrow B_{2,2}^{0, \frac{1}{2}}(\Omega)\right) \lesssim e_{k}\left(\mathrm{id}: \mathcal{N}_{u, p, q}^{s}(\Omega) \hookrightarrow \operatorname{bmo}(\Omega)\right) \\
& \quad \lesssim e_{k}\left(\operatorname{id}_{\tau}: B_{p, \infty}^{s, \tau} \hookrightarrow B_{2,2}^{0, \frac{1}{2}}(\Omega)\right)
\end{aligned}
$$

which in view of Theorem 4.2(ii) gives the desired result.

Step 2 Now we turn to the embedding id: $\mathcal{A}_{u, p, q}^{s}(\Omega) \hookrightarrow L_{\infty}(\Omega)$ and make use of the above embeddings to the $B$-scale. By (2.10) and (2.18) with $0 \leq \tau=\frac{1}{p}-\frac{1}{u}$, we have

$$
\begin{aligned}
& B_{p, \min \{p, q\}}^{s, \tau}(\Omega) \hookrightarrow \mathcal{E}_{u, p, q}^{s}(\Omega)=F_{p, q}^{s, \tau}(\Omega) \hookrightarrow B_{p, \max \{p, q\}}^{s, \tau}(\Omega) \\
& \hookrightarrow B_{\infty, 1}^{0}(\Omega) \hookrightarrow L_{\infty}(\Omega) \hookrightarrow B_{\infty, \infty}^{0}(\Omega) .
\end{aligned}
$$

According to Theorem 4.2(ii), we then get

$$
\begin{aligned}
& e_{k}\left(\mathrm{id}: \mathcal{E}_{u, p, q}^{s}(\Omega) \hookrightarrow L_{\infty}(\Omega)\right) \lesssim e_{k}\left(\operatorname{id}_{\tau}: B_{p, \max \{p, q\}}^{s, \tau}(\Omega)\right. \\
& \left.\hookrightarrow B_{\infty, 1}^{0}(\Omega)\right) \lesssim k^{-\frac{s}{d}}, \quad k \in \mathbb{N}
\end{aligned}
$$

and

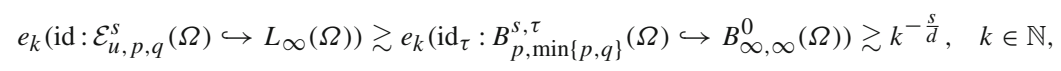

whenever $s>\frac{d}{u}$, which completes the proof for $\mathcal{A}=\mathcal{E}$.

Similarly we obtain the result for $\mathcal{A}=\mathcal{N}$. Namely, we have

$$
e_{k}\left(\operatorname{id}_{\mathcal{N}}: \mathcal{N}_{u, p, q}^{s}(\Omega) \hookrightarrow \mathcal{N}_{\infty, \infty, \infty}^{0}(\Omega)\right) \lesssim e_{k}(\mathrm{id}) \lesssim e_{k}\left(\operatorname{id}_{\mathcal{N}}: \mathcal{N}_{u, p, \infty}^{s}(\Omega) \hookrightarrow \mathcal{N}_{\infty, \infty, 1}^{0}(\Omega)\right)
$$

which in view of Theorem 4.1(ii) gives the result.

Remark 4.7 Corollary 4.6 extends some result on entropy numbers for the target space $L_{\infty}(\Omega)$ obtained in [16]. There we could only cover the case $s>\frac{d}{p}$.

In a parallel way we can further characterize the compactness of the embeddings described by Corollary 3.8.

Corollary 4.8 Let $s \in \mathbb{R}, \tau \geq 0,0<p, q \leq \infty$ (with $p<\infty$ in case of $A=F$ ), and assume $s>d\left(\frac{1}{p}-\tau\right)$. Then the entropy numbers of the compact embeddings $\operatorname{id}_{\tau}: A_{p, q}^{s, \tau}(\Omega) \hookrightarrow L_{\infty}(\Omega)$ and $\operatorname{id}_{\tau}: A_{p, q}^{s, \tau}(\Omega) \hookrightarrow \operatorname{bmo}(\Omega)$ behave like

$$
e_{k}\left(\mathrm{id}: A_{p, q}^{s, \tau}(\Omega) \hookrightarrow L_{\infty}(\Omega)\right) \sim e_{k}\left(\mathrm{id}: A_{p, q}^{s, \tau}(\Omega) \hookrightarrow \operatorname{bmo}(\Omega)\right) \sim k^{-\frac{s}{d}-\left(\tau-\frac{1}{p}\right)_{+}}, \quad k \in \mathbb{N} .
$$


Proof We proceed as above and observe that Theorem 4.2(i) is again excluded for the same reasons.

Finally we deal with the target space $L_{r}(\Omega), 1 \leq r<\infty$. First we recall our result obtained in [17, Corollary 4.8].

Corollary 4.9 Let $1 \leq r<\infty, 0<p \leq u<\infty$, or $p=u=\infty$, and $s>$ $d \frac{p}{u}\left(\frac{1}{p}-\frac{1}{r}\right)_{+}$.

(i) If $p \geq r$ and $s>0$, or $p<r$ and $s>d\left(\frac{1}{p}-\frac{1}{r}\right)$, then

$$
e_{k}\left(\operatorname{id}: \mathcal{A}_{u, p, q}^{s}(\Omega) \hookrightarrow L_{r}(\Omega)\right) \sim k^{-\frac{s}{d}}
$$

(ii) If $p<r$ and $d \frac{p}{u}\left(\frac{1}{p}-\frac{1}{r}\right)<s \leq d\left(\frac{1}{p}-\frac{1}{r}\right)$, then there exists some $c>0$ and for any $\varepsilon>0$ some $c_{\varepsilon}>0$ such that for all $k \in \mathbb{N}$,

$$
c k^{-\frac{u}{u-p}\left(\frac{s}{d}-\frac{p}{u}\left(\frac{1}{p}-\frac{1}{r}\right)\right)} \leq e_{k}\left(\mathrm{id}: \mathcal{A}_{u, p, q}^{s}(\Omega) \hookrightarrow L_{r}(\Omega)\right) \leq c_{\varepsilon} k^{-\frac{u}{u-p}\left(\frac{s}{d}-\frac{p}{u}\left(\frac{1}{p}-\frac{1}{r}\right)\right)+\varepsilon} .
$$

Now we can strengthen our above compactness result Corollary 3.10 as follows.

Corollary 4.10 Let $s \in \mathbb{R}, 0<p, q \leq \infty$ (with $p<\infty$ in case of $A=F$ ), $1 \leq r<$ $\infty, \tau>0$. Assume that (3.34) is satisfied.

(i) If

$$
\tau<\frac{1}{p}, \quad p<r, \quad \text { and }(1-p \tau)\left(\frac{1}{p}-\frac{1}{r}\right)<\frac{s}{d}<\frac{1}{p}-\frac{1}{r}
$$

then there exists some $c>0$ and for any $\varepsilon>0$ some $c_{\varepsilon}>0$ such that for all $k \in \mathbb{N}$,

$$
c k^{-\frac{1}{p \tau}\left(\frac{s}{d}-(1-p \tau)\left(\frac{1}{p}-\frac{1}{r}\right)\right)} \leq e_{k}\left(\operatorname{id}: A_{p, q}^{s, \tau}(\Omega) \hookrightarrow L_{r}(\Omega)\right) \leq c_{\varepsilon} k^{-\frac{1}{p \tau}\left(\frac{s}{d}-(1-p \tau)\left(\frac{1}{p}-\frac{1}{r}\right)\right)+\varepsilon} .
$$

(ii) In all other cases admitted by (3.34), it holds

$$
e_{k}\left(\mathrm{id}: A_{p, q}^{s, \tau}(\Omega) \hookrightarrow L_{r}(\Omega)\right) \sim k^{-\frac{s}{d}-\left(\tau-\frac{1}{p}\right)_{+}}, \quad k \in \mathbb{N} .
$$

Proof We apply Theorem 4.2 and follow otherwise exactly the same line of arguments, in particular, with $B_{r, 1}^{0}(\Omega) \hookrightarrow L_{r}(\Omega) \hookrightarrow B_{r, \infty}^{0}(\Omega)$.

Remark 4.11 Note that we (only) have an influence of $r$ when we are in the proper Morrey case $\left(0<\tau<\frac{1}{p}\right)$ and $s$ is small enough. 
Remark 4.12 Due to their similarities, we do not present the special cases when the source or the target space matches the classical spaces $B_{p, q}^{s}$ and $F_{p, q}^{s}$, that is when $\tau_{1}=0$ or $\tau_{2}=0$. However, we would like to remark that the result is not symmetric in the sense that we have different results for both cases. Namely, when $\tau_{1}=0$, part (i) of Theorem 4.2 is excluded, while for the case where $\tau_{2}=0$ both parts of the theorem are relevant, naturally with the proper adaptations for this particular case.

\section{Approximation numbers}

Finally we briefly collect some partial results about approximation numbers of the embedding $\mathrm{id}_{\tau}$, recall their definition (2.24). Now we assume that $\Omega$ is a $C^{\infty}$ domain. In [43] we obtained some first result for approximation numbers $a_{k}\left(\mathrm{id}_{\tau}\right)$ when the target space was $L_{\infty}(\Omega)$ : let $p \in[2, \infty]$ (with $p<\infty$ in the $F$-case), $q \in(0, \infty]$, $0 \leq \tau<\frac{1}{p}$ and $d\left(\frac{1}{p}-\tau\right)<s<d\left(\frac{1}{p}-\tau\right)+1$. Then

$$
a_{k}\left(\mathrm{id}: A_{p, q}^{s, \tau}(\Omega) \rightarrow L_{\infty}(\Omega)\right) \sim k^{-\frac{s}{d}-\tau+\frac{1}{p}}, \quad k \in \mathbb{N}
$$

In [16] we studied the situation for the embedding $\operatorname{id}_{\mathcal{A}}$ with the following result.

Proposition 5.1 Let $s_{i} \in \mathbb{R}, 0<q_{i} \leq \infty, 0<p_{i} \leq u_{i}<\infty$ or, in the case of $\mathcal{N}$-spaces, allow also $p_{i}=u_{i}=\infty, i=1,2$, with

$$
s_{1}>s_{2}, \text { and } p_{1} \geq u_{2} \text {. }
$$

Then

$$
a_{k}\left(\operatorname{id}_{\mathcal{A}}: \mathcal{A}_{u_{1}, p_{1}, q_{1}}^{s_{1}}(\Omega) \hookrightarrow \mathcal{A}_{u_{2}, p_{2}, q_{2}}^{s_{2}}(\Omega)\right) \sim k^{-\frac{s_{1}-s_{2}}{d}}, \quad k \in \mathbb{N} .
$$

The above proposition coincides with [16, Corollary 3.4(i)] apart from the case when $p_{i}=u_{i}=\infty$ for $i=1$ or $i=2$. But this extension can easily be verified following the short proof in [16]. We also refer to [1, Section 6] where also the periodic case and more general Morrey type spaces were studied.

Now we give some partial counterpart of Theorem 4.2 in terms of approximation numbers.

Corollary 5.2 Let $s_{i} \in \mathbb{R}, 0<q_{i} \leq \infty, 0<p_{i} \leq \infty$ (with $p_{i}<\infty$ in case of $A=F), \tau_{i} \geq 0, i=1,2$. Assume that $s_{1}-s_{2}>d \gamma\left(\tau_{1}, \tau_{2}, p_{1}, p_{2}\right)$ and, in addition,

(i) either $\tau_{1} \geq \frac{1}{p_{1}}$,

(ii) or $\tau_{i}<\frac{1}{p_{i}}$, $i=1,2$, with $s_{1}>s_{2}$ and $\tau_{2} \leq \frac{1}{p_{2}}-\frac{1}{p_{1}}$.

Then we obtain for the approximation numbers of the compact embedding

$$
\operatorname{id}_{\tau}: A_{p_{1}, q_{1}}^{s_{1}, \tau_{1}}(\Omega) \hookrightarrow A_{p_{2}, q_{2}}^{s_{2}, \tau_{2}}(\Omega)
$$

that

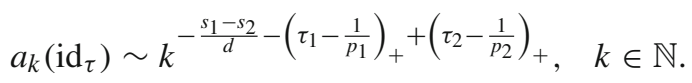


Proof Step 1 We begin with case (i) and assume that $\tau_{1} \geq \frac{1}{p_{1}}$, with $q_{1}=\infty$ when $\tau_{1}=\frac{1}{p_{1}}$. In case of $\tau_{2} \geq \frac{1}{p_{2}}$ with $q_{2}=\infty$ when $\tau_{2}=\frac{1}{p_{2}}$, then we use classical Besov space results,

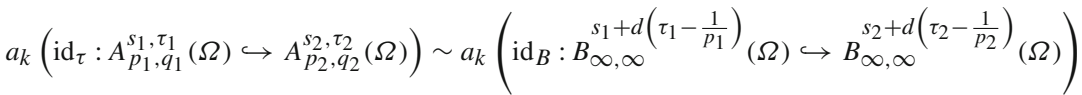

$$
\begin{aligned}
& \sim k^{-\frac{s_{1}-s_{2}}{d}-\left(\tau_{1}-\frac{1}{p_{1}}\right)+\left(\tau_{2}-\frac{1}{p_{2}}\right)}, \quad k \in \mathbb{N},
\end{aligned}
$$

in view of Proposition 2.7 and the corresponding approximation number result (2.28). This proves (5.4) in this case.

If $0 \leq \tau_{2}<\frac{1}{p_{2}}$, then we proceed similar to Substep 2.2 of the proof of Theorem 4.2 and make use of the chains of embeddings (3.20) and (3.21). Therefore, we get

$$
\begin{aligned}
a_{k}\left(\operatorname{id}_{\mathcal{N}}: \mathcal{N}_{\infty, \infty, \infty}^{s_{1}+d\left(\tau_{1}-\frac{1}{p_{1}}\right)}(\Omega) \hookrightarrow \mathcal{N}_{u_{2}, p_{2}, \infty}^{s_{2}}(\Omega)\right) & \lesssim a_{k}\left(\operatorname{id}_{\tau}\right) \\
& \lesssim a_{k}\left(\operatorname{id}: \mathcal{N}_{\infty, \infty, \infty}^{s_{1}+d\left(\tau_{1}-\frac{1}{p_{1}}\right)}(\Omega) \hookrightarrow \mathcal{A}_{u_{2}, p_{2}, q_{2}}^{s_{2}}(\Omega)\right)
\end{aligned}
$$

which, in view of Proposition 5.1, leads to

$$
a_{k}\left(\operatorname{id}_{\tau}\right) \sim k^{-\frac{s_{1}-s_{2}}{d}-\left(\tau_{1}-\frac{1}{p_{1}}\right)}, \quad k \in \mathbb{N} .
$$

As for the case $\tau_{2}=\frac{1}{p_{2}}, q_{2}<\infty$, we can follow Substep 3.4 of the proof of Theorem 4.2 and apply Proposition 5.1 instead of Theorem 4.1.

Step 2 Assume that $\tau_{1}=\frac{1}{p_{1}}$ with $q_{1}<\infty$. Again we check our above proof of Theorem 4.2 and find that Substep 3.1 with Proposition 5.1 cover the case $0<\tau_{2}<\frac{1}{p_{2}}$, while Substep 3.2 of that proof is related to the case $\tau_{2} \geq \frac{1}{p_{2}}$ with $q_{2}=\infty$ if $\tau_{2}=\frac{1}{p_{2}}$, and Substep 3.5 concerns the situation when $\tau_{2}=\frac{1}{p_{2}}$ with $q_{2}<\infty$. In the latter two cases we benefit from (2.28).

Step 3 We deal with (ii). In this case, we can apply (2.18) and Proposition 5.1 to obtain the result for $A_{p, q}^{s, \tau}=F_{p, q}^{s, \tau}$. Otherwise we argue similarly as in Step 1 and, using (3.17) and (3.18) this time, we get

$$
\begin{aligned}
& a_{k}\left(\operatorname{id}_{\mathcal{N}}: \mathcal{N}_{u_{1}, p_{1}, q_{1}}^{s_{1}}(\Omega) \hookrightarrow \mathcal{N}_{u_{2}, p_{2}, \infty}^{s_{2}}(\Omega)\right) \lesssim a_{k}\left(\operatorname{id}_{\tau}\right) \\
& \lesssim a_{k}\left(\operatorname{id}_{\mathcal{N}}: \mathcal{N}_{u_{1}, p_{1}, \infty}^{s_{1}}(\Omega) \hookrightarrow \mathcal{N}_{u_{2}, p_{2}, q_{2}}^{s_{2}}(\Omega)\right),
\end{aligned}
$$

where $\frac{1}{u_{i}}=\frac{1}{p_{i}}-\tau_{i}, i=1,2$, as usual. Now we apply Proposition 5.1 (with $\mathcal{A}=\mathcal{N}$ ), under the additional assumptions made in (ii), and benefit again from the independence of $q_{i}$. 
Remark 5.3 It is obvious from the above proof, in particular in Steps 1 and 2, that we could follow all the arguments of the proof of Theorem 4.2 to deal with the remaining cases. However, in view of the additional restriction (5.2) in Proposition 5.1 and the observation of $p$-dependence in (2.28) this leads to partial one-sided results only.

Remark 5.4 Note that one could reformulate Corollary 5.2 in terms of the hybrid spaces $L^{r} A_{p, q}^{s}(\Omega)$ in the spirit of Remark 4.3. We leave it to the reader.

Finally we conclude a few special cases from Corollary 5.2 and start with the case $\tau_{1}=\tau_{2}=\tau$.

Corollary 5.5 Let $s_{i} \in \mathbb{R}, 0<q_{i} \leq \infty, 0<p_{i} \leq \infty$ (with $p_{i}<\infty$ in case of $A=F), i=1,2$, and $\tau \geq 0$. Assume that (3.33) is satisfied and, in addition,

(i) either $\tau \geq \frac{1}{p_{1}}$,

(ii) or $\tau<\min \left\{\frac{1}{p_{1}}, \frac{1}{p_{2}}\right\}$, with $\tau \leq \frac{1}{p_{2}}-\frac{1}{p_{1}}$.

Then we obtain for the approximation numbers of the compact embedding

$$
\operatorname{id}_{\tau}: A_{p_{1}, q_{1}}^{s_{1}, \tau}(\Omega) \hookrightarrow A_{p_{2}, q_{2}}^{s_{2}, \tau}(\Omega)
$$

that

$$
a_{k}\left(\mathrm{id}_{\tau}\right) \sim\left\{\begin{array}{ll}
k^{-\frac{s_{1}-s_{2}}{d}+\frac{1}{p_{1}}-\frac{1}{p_{2}}}, & \text { if } \tau \geq \max \left\{\frac{1}{p_{1}}, \frac{1}{p_{2}}\right\}, \\
k^{-\frac{s_{1}-s_{2}}{d}-\tau+\frac{1}{p_{1}}}, & \text { if } \frac{1}{p_{2}}>\tau \geq \frac{1}{p_{1}}, \\
k^{-\frac{s_{1}-s_{2}}{d}}, & \text { if } \tau<\min \left\{\frac{1}{p_{1}}, \frac{1}{p_{2}}\right\}
\end{array} \text { and } \tau \leq \frac{1}{p_{2}}-\frac{1}{p_{1}} .\right.
$$

Next we consider the special target spaces $L_{r}(\Omega), 1 \leq r \leq \infty$, and $\operatorname{bmo}(\Omega)$.

Corollary 5.6 Let $s \in \mathbb{R}, 0<p, q \leq \infty$ (with $p<\infty$ in case of $A=F$ ), and assume that $\tau \geq \frac{1}{p}$ and $s>d\left(\frac{1}{p}-\tau\right)$. Then the approximation numbers of the compact embeddings id : $A_{p, q}^{s, \tau}(\Omega) \hookrightarrow L_{\infty}(\Omega)$ and $\mathrm{id}: A_{p, q}^{s, \tau}(\Omega) \hookrightarrow \operatorname{bmo}(\Omega)$ behave like $a_{k}\left(\mathrm{id}: A_{p, q}^{s, \tau}(\Omega) \hookrightarrow L_{\infty}(\Omega)\right) \sim a_{k}\left(\mathrm{id}: A_{p, q}^{s, \tau}(\Omega) \hookrightarrow \operatorname{bmo}(\Omega)\right) \sim k^{-\frac{s}{d}-\tau+\frac{1}{p}}, \quad k \in \mathbb{N}$.

Remark 5.7 In our paper [43] we obtained already that

$$
a_{k}\left(\mathrm{id}: A_{p, q}^{s, \tau}(\Omega) \hookrightarrow L_{\infty}(\Omega)\right) \sim k^{-\frac{s}{d}-\tau+\frac{1}{p}}, \quad k \in \mathbb{N},
$$

if $0<p, q \leq \infty$ (with $p<\infty$ if $A=F),\left(\frac{1}{p}-\frac{1}{2}\right)_{+} \leq \tau<\frac{1}{p}$, and $d\left(\frac{1}{p}-\tau\right)<s<$ $d\left(\frac{1}{p}-\tau\right)+1$ (please note the misprints in [43, Corollary 5.10]). So the above result can be seen as some partial extension to the case when $\tau \geq \frac{1}{p}$.

The partial counterpart of Corollary 4.10 reads as follows. 
Corollary 5.8 Let $s \in \mathbb{R}, 0<p, q \leq \infty$ (with $p<\infty$ in case of $A=F), 1 \leq r<\infty$, $\tau \geq 0$. Assume that (3.34) is satisfied and, in addition, that

(i) either $\tau \geq \frac{1}{p}$,

(ii) or $\tau<\frac{1}{p} \leq \frac{1}{r}$.

\section{Then we obtain that}

$$
a_{k}\left(\mathrm{id}: A_{p, q}^{s, \tau}(\Omega) \hookrightarrow L_{r}(\Omega)\right) \sim k^{-\frac{s}{d}-\left(\tau-\frac{1}{p}\right)_{+}}, \quad k \in \mathbb{N} .
$$

Remark 5.9 Note that Corollaries 5.6 and 5.8 deal with situations where entropy and approximation numbers show the same asymptotic behaviour, cf. Corollaries 4.8 and 4.10, which is in general not the case.

Acknowledgements Open Access funding provided by Projekt DEAL. Open Access funding provided by Projekt DEAL.

Open Access This article is licensed under a Creative Commons Attribution 4.0 International License, which permits use, sharing, adaptation, distribution and reproduction in any medium or format, as long as you give appropriate credit to the original author(s) and the source, provide a link to the Creative Commons licence, and indicate if changes were made. The images or other third party material in this article are included in the article's Creative Commons licence, unless indicated otherwise in a credit line to the material. If material is not included in the article's Creative Commons licence and your intended use is not permitted by statutory regulation or exceeds the permitted use, you will need to obtain permission directly from the copyright holder. To view a copy of this licence, visit http://creativecommons.org/licenses/by/4.0/.

\section{References}

1. Baituyakova, Z., Sickel, W.: Strong summability of Fourier series and generalized Morrey spaces. Anal. Math. 43(3), 371-414 (2017)

2. Caetano, A.: About approximation numbers in function spaces. J. Approx. Theory 94, 383-395 (1998)

3. Carl, B., Stephani, I.: Entropy, Compactness and the Approximation of Operators. Cambridge University Press, Cambridge (1990)

4. Edmunds, D.E., Evans, W.D.: Spectral Theory and Differential Operators. Clarendon Press, Oxford (1987)

5. Edmunds, D.E., Triebel, H.: Entropy numbers and approximation numbers in function spaces. Proc. Lond. Math. Soc. 58(3), 137-152 (1989)

6. Edmunds, D.E., Triebel, H.: Entropy numbers and approximation numbers in function spaces II. Proc. Lond. Math. Soc. 64(3), 153-169 (1992)

7. Edmunds, D.E., Triebel, H.: Function Spaces, Entropy Numbers, Differential Operators. Cambridge University Press, Cambridge (1996)

8. El Baraka, A.: An embedding theorem for Campanato spaces. Electron. J. Differ. Equ. 66, 1-17 (2002)

9. El Baraka, A.: Function spaces of BMO and Campanato type. In: Proceedings of the 2002 Fez Conference on Partial Differential Equations, 109-115 (electronic), Electron. J. Differ. Equ. Conf. 9, Southwest Texas State University, San Marcos, TX (2002)

10. El Baraka, A.: Littlewood-Paley characterization for Campanato spaces. J. Funct. Spaces Appl. 4, 193-220 (2006)

11. Haroske, D.D., Moura, S.D., Schneider, C., Skrzypczak, L.: Unboundedness properties of smoothness Morrey spaces of regular distributions on domains. Sci. China Math. 60(12), 2349-2376 (2017)

12. Haroske, D.D., Moura, S.D., Skrzypczak, L.: Smoothness Morrey Spaces of regular distributions, and some unboundedness properties. Nonlinear Anal. Ser. A Theory Methods Appl. 139, 218-244 (2016)

13. Haroske, D.D., Skrzypczak, L.: Continuous embeddings of Besov-Morrey function spaces. Acta Math. Sin. (Engl. Ser.) 28, 1307-1328 (2012) 
14. Haroske, D.D., Skrzypczak, L.: Embeddings of Besov-Morrey spaces on bounded domains. Stud. Math. 218, 119-144 (2013)

15. Haroske, D.D., Skrzypczak, L.: On Sobolev and Franke-Jawerth embeddings of smoothness Morrey spaces. Rev. Mat. Complut. 27, 541-573 (2014)

16. Haroske, D.D., Skrzypczak, L.: Some quantitative result on compact embeddings in smoothness Morrey spaces on bounded domains; an approach via interpolation. In: Function Spaces XII, Volume 119 of Banach Center Publications, pp. 181-191. Polish Academy of Sciencesi, Warsaw (2019)

17. Haroske, D.D., Skrzypczak, L.: Entropy numbers of compact embeddings of smoothness Morrey spaces on bounded domains. J. Approx. Theory 256, 105424 (2020)

18. König, H.: Eigenvalue Distribution of Compact Operators. Birkhäuser, Basel (1986)

19. Kozono, H., Yamazaki, M.: Semilinear heat equations and the Navier-Stokes equation with distributions in new function spaces as initial data. Commun. Partial Differ. Equ. 19, 959-1014 (1994)

20. Li, P., Xia, J., Yang, Q.: Global mild solutions to modified Navier-Stokes equations with small initial data in critical Besov- $Q$ spaces. Electron. J. Differ. Equ. 2014, No. 185

21. Mazzucato, A.L.: Besov-Morrey spaces: function space theory and applications to non-linear PDE. Trans. Am. Math. Soc. 355, 1297-1364 (2003)

22. Morrey, C.B.: On the solutions of quasi-linear elliptic partial differential equations. Trans. Am. Math. Soc. 43, 126-166 (1938)

23. Peetre, J.: On the theory of $\mathcal{L}_{p, \lambda}$ spaces. J. Funct. Anal. 4, 71-87 (1969)

24. Pietsch, A.: Operator Ideals. North-Holland, Amsterdam (1980)

25. Pietsch, A.: Eigenvalues and $s$-Numbers. Akad. Verlagsgesellschaft Geest \& Portig, Leipzig (1987)

26. Rosenthal, M.: Local means, wavelet bases, representations, and isomorphisms in Besov-Morrey and Triebel-Lizorkin-Morrey spaces. Math. Nachr. 286, 59-87 (2013)

27. Sawano, Y.: Wavelet characterizations of Besov-Morrey and Triebel-Lizorkin-Morrey spaces. Funct. Approx. Comment. Math. 38, 93-107 (2008)

28. Sawano, Y.: A note on Besov-Morrey spaces and Triebel-Lizorkin-Morrey spaces. Acta Math. Sin. (Engl. Ser.) 25, 1223-1242 (2009)

29. Sawano, Y., Tanaka, H.: Decompositions of Besov-Morrey spaces and Triebel-Lizorkin-Morrey spaces. Math. Z. 257, 871-905 (2007)

30. Sawano, Y., Tanaka, H.: Besov-Morrey spaces and Triebel-Lizorkin-Morrey spaces for non-doubling measures. Math. Nachr. 282, 1788-1810 (2009)

31. Sawano, Y.: Besov-Morrey spaces and Triebel-Lizorkin-Morrey spaces on domains. Math. Nachr. 283(10), 1456-1487 (2010)

32. Sickel, W.: Smoothness spaces related to Morrey spaces-a survey. I. Eurasian Math. J. 3, 110-149 (2012)

33. Sickel, W.: Smoothness spaces related to Morrey spaces-a survey. II. Eurasian Math. J. 4, 82-124 (2013)

34. Tang, L., Xu, J.: Some properties of Morrey type Besov-Triebel spaces. Math. Nachr. 278, 904-917 (2005)

35. Triebel, H.: Theory of Function Spaces. Birkhäuser, Basel (1983)

36. Triebel, H.: Theory of Function Spaces. II. Birkhäuser, Basel (1992)

37. Triebel, H.: Theory of Function Spaces. III. Birkhäuser, Basel (2006)

38. Triebel, H.: Local Function Spaces, Heat and Navier-Stokes Equations. EMS Tracts in Mathematics, vol. 20, European Mathematical Society (EMS), Zürich (2013)

39. Triebel, H.: Hybrid Function Spaces, Heat and Navier-Stokes Equations. EMS Tracts in Mathematics, vol. 24. European Mathematical Society (EMS), Zürich (2015)

40. Yang, D., Yuan, W.: A new class of function spaces connecting Triebel-Lizorkin spaces and Q spaces. J. Funct. Anal. 255, 2760-2809 (2008)

41. Yang, D., Yuan, W.: New Besov-type spaces and Triebel-Lizorkin-type spaces including Q spaces. Math. Z. 265, 451-480 (2010)

42. Yang, D., Yuan, W.: Relations among Besov-type spaces, Triebel-Lizorkin-type spaces and generalized Carleson measure spaces. Appl. Anal. 92, 549-561 (2013)

43. Yuan, W., Haroske, D.D., Moura, S.D., Skrzypczak, L., Yang, D.: Limiting embeddings in smoothness Morrey spaces, continuity envelopes and applications. J. Approx. Theory 192, 306-335 (2015)

44. Yuan, W., Haroske, D.D., Skrzypczak, L., Yang, D.: Embedding properties of Besov-type spaces. Appl. Anal. 94(2), 318-340 (2015) 
45. Yuan, W., Sickel, W., Yang, D.: Morrey and Campanato Meet Besov, Lizorkin and Triebel, Lecture Notes in Mathematics, vol. 2005. Springer, Berlin (2010)

46. Yuan, W., Sickel, W., Yang, D.: On the coincidence of certain approaches to smoothness spaces related to Morrey spaces. Math. Nachr. 286, 1571-1584 (2013)

Publisher's Note Springer Nature remains neutral with regard to jurisdictional claims in published maps and institutional affiliations. 